\title{
Topología de la arqueología litúrgica del primer románico del Val d'Aran
}

\section{Topology of liturgical archeology of the first Romanesque of Val d'Aran}

\author{
Josep Lluis i Ginovart ${ }^{1}$, Mónica López Piquer² \\ Universitat Internacional de Catalunya \\ Sergio Coll Pla ${ }^{3}$, Agustí Costa Jover ${ }^{4}$ \\ Universitat Rovira i Virgili
}

\begin{abstract}
RESUMEN
En el Val d'Aran existe un conjunto de iglesias románicas construidas entre el siglo XI y el primer tercio del XIII, de lo que se ha llamado primer románico aranés. Estas iglesias han sido sistemáticamente estudiadas a través de la captura masiva de datos con un Escáner Láser Terrestre (TLS) en cinco campañas (2014-2015). Los datos obtenidos nos han permitido hacer un análisis transversal de estas iglesias con el fin de analizar su topología y sus relaciones y asonancias desde la aproximación a las liturgias románicas, especialmente desde el Gemma animae de Honorio de Autun y el Mitralis de Officio de Sicardo, obispo de Cremona. Para ello, se determina la relación entre el espacio sagrado, el Sancta, el lugar de los fieles, y el Sancta Sanctorum. La relación de las características formales, superficies, volúmenes, proporciones y las orientaciones de las iglesias, nos acercan a su conocimiento epistemológico.
\end{abstract}

Palabras clave: románico; Val d’Aran; Honorio de Autun; Sicardo obispo de Cremona; topología.

\begin{abstract}
In Val d'Aran there is a group of Romanesque churches built between the 11th and the first third of the 13th century, of what has been called the first Aranese Romanesque. T hese churches have b een systematically studied through mass data capture with a Terrestrial Laser Scanner (TLS) in five times (2014-2015). The data obtained have allowed us to make a transversal analysis of these churches in order to analyse the topology of them and their relations and assonances from the approach to the Romanesque liturgies, especially from the Gemma animae of Honorius of Autun and the Mitralis de Officio of Sicard, Cremona's bishop. For this purpose, the relationship to the sacred space, the Sancta, in place of the people, and the so-called Sancta Sanctorum, where the priest officiates, is studied, as well as the relation of areas, volumes and orientations of these churches.
\end{abstract}

Key words: Romanesque; Val d'Aran; Honorius of Autun; Sicard Cremona's Bishop; topology.

Recibido: 13-02-2017. Aceptado: 30-08-2017. Publicado online: 12-12-2017

Cómo citar este artículo / Citation

Lluis i Ginovart, J., López Piquer, M., Coll Pla, S. y Costa Jover, A. 2017: "Topología de la arqueología litúrgica del primer románico del Val d’Aran", Arqueología de la Arquitectura, 14: e059. doi: http://dx.doi.org/10.3989/arq.arqt.2017.013.

\section{Copyright}

(C) 2017 CSIC. Este es un artículo de acceso abierto distribuido bajo los términos de una licencia de uso y distribución Creative Commons Attribution (CC-by) España 3.0.

\footnotetext{
jlluis@uic.es / ORCID iD: http://orcid.org/0000-0001-5957-762X.

mlopezp@uic.es / ORCID iD: http://orcid.org/0000-0002-3945-5462.

sergio.coll@urv.cat / ORCID iD: http://orcid.org/0000-0002-4718-5810.

agusti.costa@urv.cat / ORCID iD: http://orcid.org/0000-0002-6194-3243.
} 


\section{INTRODUCCIÓN}

El Val d'Aran conserva treinta y cinco iglesias en pie, o con restos suficientemente significativos, de cuya construcción se tiene una cronología algo incierta, pero iniciada a partir del siglo XI. Las primeras construcciones del Val, se extienden hasta el siglo XIII, cuando todavía no habían hecho su aparición los elementos característicos del arte gótico. Este periodo es definido tradicionalmente como románico aranés. Las construcciones del románico se realizaron bajo la influencia de las principales liturgias de la época, según exponen diversos autores como José Antonio Íñiguez Herrero (1986), Santiago Sebastián (1994), Nicolas Reveyron (2003), Francesca Mambelli (2004), Constant J. Mews (2009), Eduardo Carrero (2009). Estas liturgias se basaban en el Liber officialis (820-826) de Amalario de Metz (c. 780-851), que inspirará a los principales autores de los siglos románicos: Ruperto de Deutz (c. 1075-1129), con su Liber de divinis officiis (1120), el Gemma animae (c. 1120) de Honorio de Autun (1080-c. 1153), el Mitralis de Officio (1190) de Sicardo obispo de Cremona (1185-1215), Jean Beleth (fl. 1135-1182) con su Rationale divinorum officiorum, (c. 1150), Innocenzo III (1161-1216) con De sacro altaris mysterio (c. 1200) y, finalmente, el Prochiron, vulgo rationale divinorum officiorum (1291) de Guillermo de Durando (1230-1296) de mayor influencia en el mundo gótico.

Así, las primeras iglesias aranesas se forjan en el contexto románico con este entorno litúrgico. Además, Josep Puig i Cadafalch (1867-1956) les atribuye una conexión lombarda (Puig i Cadafalch 1906). Esta consideración difiere de aquellas iglesias ibéricas donde el rito mozárabe planteará unos postulados para su definición arquitectónica muy diferentes, ya que estos fueron paulatinamente adaptándose a los ritos europeos (Martínez 2002).

El grupo más antiguo de estas iglesias se inscribe entre los siglos XI y XII, al que se define como primer románico. Puig i Cadafalch lo define como una primera escuela de tradición foránea que será sustituida por otra de readaptación local (Puig i Cadafalch et al. 1918). Esta caracterización la hará patente Marcel Durliat (1917-2006) (Durliat y Allègre 1969), aunque la primera gran catalogación del románico aranés la realiza José Serrate Forga, bajo una forma territorial, Naut-Aran (Sarrate i Forga 1975a), MigAran (Sarrate i Forga 1975b) y Baix-Aran (Sarrate i Forga 1976a). Este primer inventario concluirá con el Esquema del Arte Románico Aranés (Sarrate i Forga 1976b), el cual ha alcanzado un reconocimiento enciclopédico en la Catalunya Romànica (Pladevall 1987).
Las iglesias que pertenecen total o parcialmente al primer románico, según Emmanuel Garland (2012) y Elisa Ros Barbosa (2015), son: Santa Maria de Cap d'Aran, Sant Estèue de Tredòs, Santa Eulària d'Unha, la nave de Sant Pèir d'Escunhau, el ábside y los restos de parte de los muros de Santa Maria de Mijaran, Sant Joan d'Arròs, el muro norte del presbiterio y parte central del ábside de Sant Ròc de Begòs, Sant Miquèu de Vilamòs, Sant Fabian d'Arres de Jos, la torre campanario de Era Mair de Diu dera Purificación de Bossòst, Sant Pèir de Betlan y la cabecera de lo que fue la antigua iglesia de Sant Blas de Vilac, actualmente reconvertida en vivienda, así como también las naves y la torre campanario de Santa Maria de Vilamòs.

\section{OBJETIVO}

El objetivo del estudio es determinar algunos aspectos relacionados con la topología de estas primitivas iglesias del Val d'Aran, entendidas como el lugar que ocupan estas construcciones. Para ello, se va a analizar la topología de estos De aedificiis sacris en los términos de los enciclopedistas medievales como Isidoro de Sevilla (c. 556-636), en el Originum sive etymologiarum libri viginti (c. 630) (Etymo. L XV. iii, iv) (Isidoro de Sevilla 1919) y Rabano Mauro (c. 776-856) en su De Universo libri viginti duo (c. 844) (Rabanus Maurus 1864), (Uni. L XIV. XXI). El objetivo es dar a conocer unas primeras conclusiones sobre las principales liturgias de la época (XI-XIII), en base al tratamiento inicial de los datos obtenidos en las cinco campañas de levantamiento (20142015). El estudio así se realiza a través de sus atributos físicos, los cuales permiten identificar las coincidencias y disonancias entre estas construcciones y las principales liturgias de referencia. Se estudia así la cantidad y calidad del espacio a través del volumen. No forman parte de la investigación algunos patrones generales sobre la proporcionalidad de estas edificaciones, dado que los patrones metrológicos no son en este momento concluyentes, y relacionados con la dimensión del Galin Reiau.

\section{METODOLOGÍA}

Se establece una metodología por comparación entre la realidad física de los edificios y las prescripciones de las liturgias románicas de Honorio de Autun, el Gemma animae (c. 1120), el Rationale divinorum officiorum, (c. 
1150) de Jean Beleth y el Mitralis de Officio (1190) de Sicardo obispo de Cremona, para poder determinar si estas edificaciones siguen o no los patrones determinados por las principales liturgias.

Así, el estudio comparado de la topología con las liturgias quiere determinar el posible seguimiento de los preceptos invocados, organizados bajo la secuencia de las decisiones a tomar para la construcción de los espacios sagrados. La primera dimensión del espacio sagrado se determina tipológicamente como Templi y De capellis. En segundo lugar, se determina la orientación, con el objetivo de identificar si siguen la orientación canónica del Ortum solis aequinoctialem. En tercer lugar, se traza el edificio con la definición del espacio sagrado, diferenciando entre el Sancta y el Sancta Sanctorum, para finalmente determinar la construcción del espacio sagrado, Muri lapidum caementati sunt religiosorum.

Para determinar el espacio topológico se ha utilizado un Escáner Láser Terrestre (Terrestrial Laser Scanner, TLS), el cual permite obtener una topografía completa de forma no invasiva y sin contacto. Se obtiene así una información geométrica y radiométrica de una superficie (Pesci et al. 2012), de gran precisión para el estudio de estos edificios (Guarnieri, Vettore y Remondino 2004). El escáner láser utilizado es el P20 de Leica, la información obtenida ha de ser tratada informáticamente, para convertirse en una nube de puntos completa mediante el programa Cyclone. Posteriormente se genera la volumetría del objeto mediante una malla tridimensional, con el programa 3DReshaper. ${ }^{5} \mathrm{La}$ precisión estimada del conjunto de puntos obtenidos de este proceso lo determinamos con un error de [0.02-0.01 m] (Fig. 1).

Teniendo en cuenta la condición topológica de los espacios sagrados, es necesario precisar las relaciones dimensionales de estas edificaciones, a través tanto del volumen como de su superficie, con respecto a la ocupación de los fieles a los que pertenece la iglesia. La importancia del dato demográfico para realizar estos cálculos ha de extrapolarse a la fecha de cuando Juan II (1267-1327) recupera el Val d'Aran y otorga los privilegios de la Era Querimònia (1313). En estos momentos se plantea que, en el Val d'Aran, había unos 7.640 habitantes (Reglà 1948). Existen otros censos posteriores como el de 1716 con 3.076 habitantes, y el de 1787 con 5.625 habitantes (Sanllehy i Sabi 1996). El Diccionario Geográfico-Estadístico-Histórico de España y sus posesiones de Ultramar (1845) de Pascual Madoz (18061870), censa 7.345 almas (Madoz 1845). Para el cálculo se utiliza el criterio de los focs u hospicia, considerando 5,2 habitantes por cada uno de ellos (Reglà 1951). Se utiliza el criterio estadístico de máxima población posible, arrojando una población a principios del siglo XIV, de 8.455 habitantes (Tabla 1).

\footnotetext{
5 La distancia promedio de puntos es de $5 \mathrm{~cm}$ y se establece un tamaño de triángulo para la detección de orificios de $10 \mathrm{~cm}$.
}

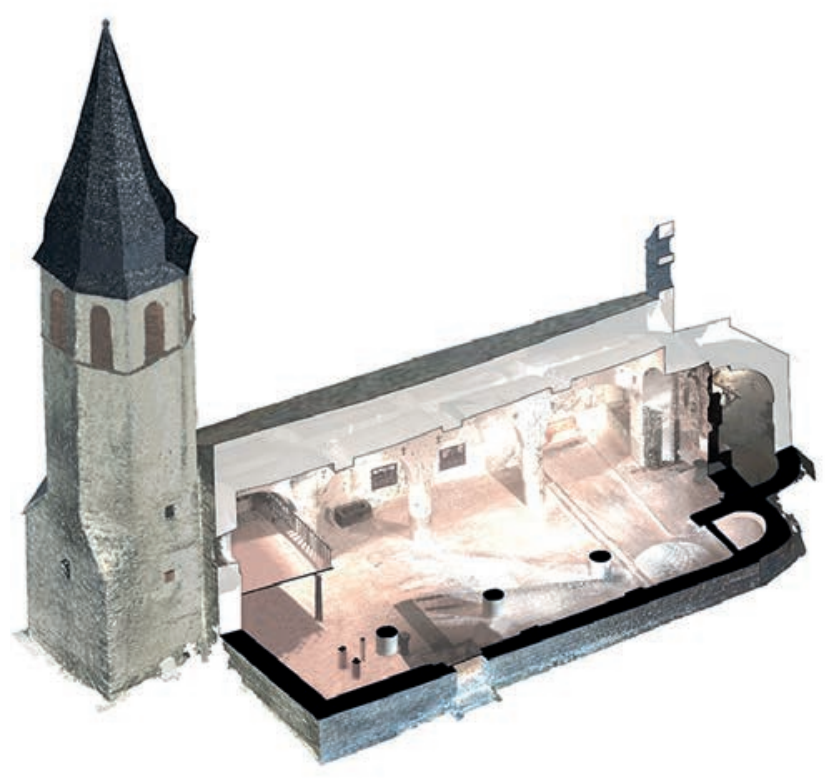

Fig. 1. Representación 3D de la toma de datos escáner láser terrestre (TLS) de Santa Eulària d'Unha (XIII). 


\begin{tabular}{|c|c|c|c|c|c|}
\hline Municipio & Otras denominaciones & Nombre iglesia & $\begin{array}{l}\text { Focs/ } \\
\text { Hospicia }\end{array}$ & Cálculo & Fieles \\
\hline Cap d'Aran & Capdaran, Capite Aranni; Caput Aranno & - & 14 & 14 & 73 \\
\hline- & Muntros & - & - & - & - \\
\hline Tredós & Trados, Tresdors, Transdosio, Trasdos & Santa Maria de Cap d'Aran & $63-60$ & 63 & 328 \\
\hline Tredós & Trados, Tresdors, Transdosio, Trasdos & Sant Estèue de Tredòs & $63-60$ & 63 & 328 \\
\hline Unha & Huyan, Unia, Hoia, Unha, Hunya & Santa Eulàlia d'Unha & $34-30$ & 34 & 177 \\
\hline Bagergue & Bayri, Bageli, Bagirio, Bagiri & Sant Fèlix de Bagergue & 55 & 55 & 286 \\
\hline Pujo & Podio, Puig, Pug, Vuert, Vuer & - & $32-30$ & 32 & 166 \\
\hline Salardú & Saverduno, Salarduno, Salardunum & Sant Andrèu de Salardú & $102-100$ & 102 & 530 \\
\hline Gessa & Hyexa, Xessa, Guixa, Gricha & Sant Pèir de Gessa & 60 & 60 & 312 \\
\hline Arties & Artis, Arters, Dartiers, Artes & Santa Maria d'Arties & $121-120$ & 121 & 629 \\
\hline Arties & Artis, Arters, Dartiers, Artes & Sant Jaume d'Arties & $121-120$ & 121 & 629 \\
\hline- & Laspan, Lespa, Espan, Lasian, Laspanno & - & $23-20$ & 23 & 120 \\
\hline Garòs & Garos, Galos & Sant Julian de Garòs & $45-50$ & 50 & 260 \\
\hline Casarilh & Canarill, Casaril, Casarihl, Casarillo & Sant Martí de Casarilh & $37-40$ & 40 & 208 \\
\hline Escunhau & $\begin{array}{l}\text { Escuñau, Aczcinia, Espinyau, Scunya, Scunhall, Scun- } \\
\text { hallbus }\end{array}$ & Sant Pèir d'Escunhau & $41-40$ & 41 & 213 \\
\hline- & Castello, Castell, Castel & - & 11 & 11 & 57 \\
\hline Betren & Berten, Bertre, Vetrenio, Bertren & Sant Estèue de Betren & $36-50$ & 50 & 260 \\
\hline Vielha & Vilabella, Vetula, Veiha, Vieilla, Biella & Sant Miquèu de Vielha & $150-160$ & 160 & 832 \\
\hline Vielha & Vilabella, Vetula, Veiha, Vieilla, Biella & Santa Maria de Mijaran & $150-160$ & 160 & 832 \\
\hline Gausac & Gausat, Gausaco, Gahusaco & Sant Martín de Tours de Gausac & $35-40$ & 40 & 208 \\
\hline Casau & Casal, Casalibus, Casalius & Sant Andrèu de Casau & $21-30$ & 30 & 156 \\
\hline Vilac & Vilac, Vilaco, Villacho, Vilacho & Sant Pèir de Vilac & $106-120$ & 120 & 624 \\
\hline Vilac & Vilac, Vilaco, Villacho, Vilacho & Sant Fèlix de Vilac & $106-120$ & 120 & 624 \\
\hline- & $\begin{array}{l}\text { Sancto Germerio et Idem }\{\text { Sta Gema }] \text {, Sangermos e Ide } \\
\text { [Sta. Gema }]\end{array}$ & - & - & - & - \\
\hline Aubèrt & Hubeit, Averto, Onert, Onerto, Olocón & Mare de Diu deth Rosèr & 40 & 40 & 208 \\
\hline Aubèrt & Hubeit, Averto, Onert, Onerto, Olocón & Sant Martín d'Aubèrt & 40 & 40 & 208 \\
\hline Betlan & Bella, Bela, Bellano, Venlano & Sant Pèir de Betlan & 30 & 30 & 156 \\
\hline Vila & Castri de Vila, Villa, Vila & - & $38-45$ & 45 & 234 \\
\hline Arròs & Aros, Arres & Sant Joan d'Arròs e Vila & $32-40$ & 40 & 208 \\
\hline Arròs & Aros, Arres & Santa Eulàlia & & & \\
\hline Montcorbau & Montcarball, Montecorballo, Muntarbal & Sant Estèue de Montcorbau & $30-30$ & 30 & 156 \\
\hline Mont & Munt, Monte & Sant Laurenç de Mont & $22-30$ & 30 & 156 \\
\hline- & Sancto Vincentio & - & - & - & - \\
\hline Vilamòs & Bilamos, Vilamos, Villamos & Santa Maria de Vilamòs & 40 & 40 & 208 \\
\hline Vilamòs & Bilamos, Vilamos, Villamos & Sant Miquèu de Vilamòs & 40 & 40 & 208 \\
\hline Benòs & Benos & Sant Martín de Benòs & 20 & 20 & 104 \\
\hline Begòs & Begos, Bergons, Begons & Sant Ròc de Begòs & 20 & 20 & 104 \\
\hline Arró & Arro, Derror, Ror, Error, Baor, Ro & Sant Martín de Arró & 10 & 10 & 52 \\
\hline- & Sentels & - & - & - & - \\
\hline Arres de Jos & $\begin{array}{l}\text { Arres, Darres , Arres Sotiras (de jos) e Arres Sobiras (de } \\
\text { sus) }\end{array}$ & Sant Fabian de Arres de Jos & 40 & 40 & 208 \\
\hline Bossòst & Bossost, Belsost, Bellsost, Bolsost, Bolson & Era Mair de Diu dera Purifacion & $70-80$ & 80 & 416 \\
\hline Les & Les, Lees, Lenes, Lens & Sant Blai de Les & $90-80$ & 90 & 468 \\
\hline Bausen & Bausen, Balsen, Bolson & Sant Pèir ad Víncula de Bausen & $25-30$ & 30 & 156 \\
\hline Canejan & Canejan, Caneia, Caneiano, Canigano & Sant Joan de Toran & $35-30$ & 35 & 182 \\
\hline Canejan & Canejan, Caneia, Caneiano, Canigano & Sant Sernilh & $35-30$ & 35 & 182 \\
\hline
\end{tabular}




\section{TOMA DE DATOS EN LAS IGLESIAS ROMÁNICAS DEL VAL D'ARAN}

La técnica de levantamiento topográfico mediante el escáner láser terrestre (TLS), es mucho más precisa que las que utilizaron los grandes historiadores de la construcción, como Eugène Emmanuel Viollet-leDuc (1814-1879) en la Eglise de Bosost (Español 2013), Lluís Domènech i Montaner (1850-1923), quien realiza los primeros levantamientos topográficos con la Escuela de Arquitectura (1905) (Granell y Ramon 2006), o Josep Puig i Cadafalch en la expedición científica al Val d'Aran y la Ribagorza (1907) (Alcolea 2008). Los métodos topográficos tradicionales fueron también utilizados por Juan Bassegoda Nonell (1930-2012) en Santa Maria d'Arties (Sàez-Aragonès 1976), y a partir de medianos de los años 80 fueron utilizados los sistemas Computer-Aided Design (CAD) en Santa María de Arties (Polo y Cots 2009).

Estas técnicas tradicionales basadas en la inspección visual, disponen de un error relativo de [3\%-5\%], mientras que las de instrumental topográfico tradicional un $1 \%$. Otra técnica fue la utilizada por la Escuela Técnica Superior de Arquitectura de la Universidad Rovira i Virgili (2012-2016), mediante levantamiento con medidas directas (Lluis i Ginovart, Costa y Coll 2016a) y fotogrametría digital (Lluis i Ginovart, Costa y Coll 2016b), basada en la técnica Close Range Photogrammetry (CRP) y la basada en algoritmos de Structure From Motion (SFM), con un error [0.05-0.03 m] (Fig. 2).

Estas primeras experiencias, permitieron identificar las limitaciones de las diferentes técnicas. Así, se decidió realizar un topografiado masivo con un dispositivo láser terrestre de todas las iglesias del Val d'Aran, a través de cinco campañas en el periodo (2014-2015) (Figs. 3, 4 y 5).

Para conseguir una representación bidimensional de estos edificios, se genera la sección longitudinal a través del plano $(\tau)$, el cual es perpendicular al plano del suelo y pasa por los puntos $\left(\mathrm{s}_{1}\right)$ y $\left(\mathrm{s}_{2}\right)$, situados en el punto más alto de la boca del presbiterio y de la fachada opuesta respectivamente. Esta sección determina un eje imaginario que marca la orientación del edificio, y permite también identificar los puntos importantes para representar la planta, con cuatro planos horizontales característicos de las posibles secciones de la planta. El primer plano horizontal es el que suponemos como indeformable $\left(\mathrm{A}_{1}\right)$, y se define a partir de la intersección

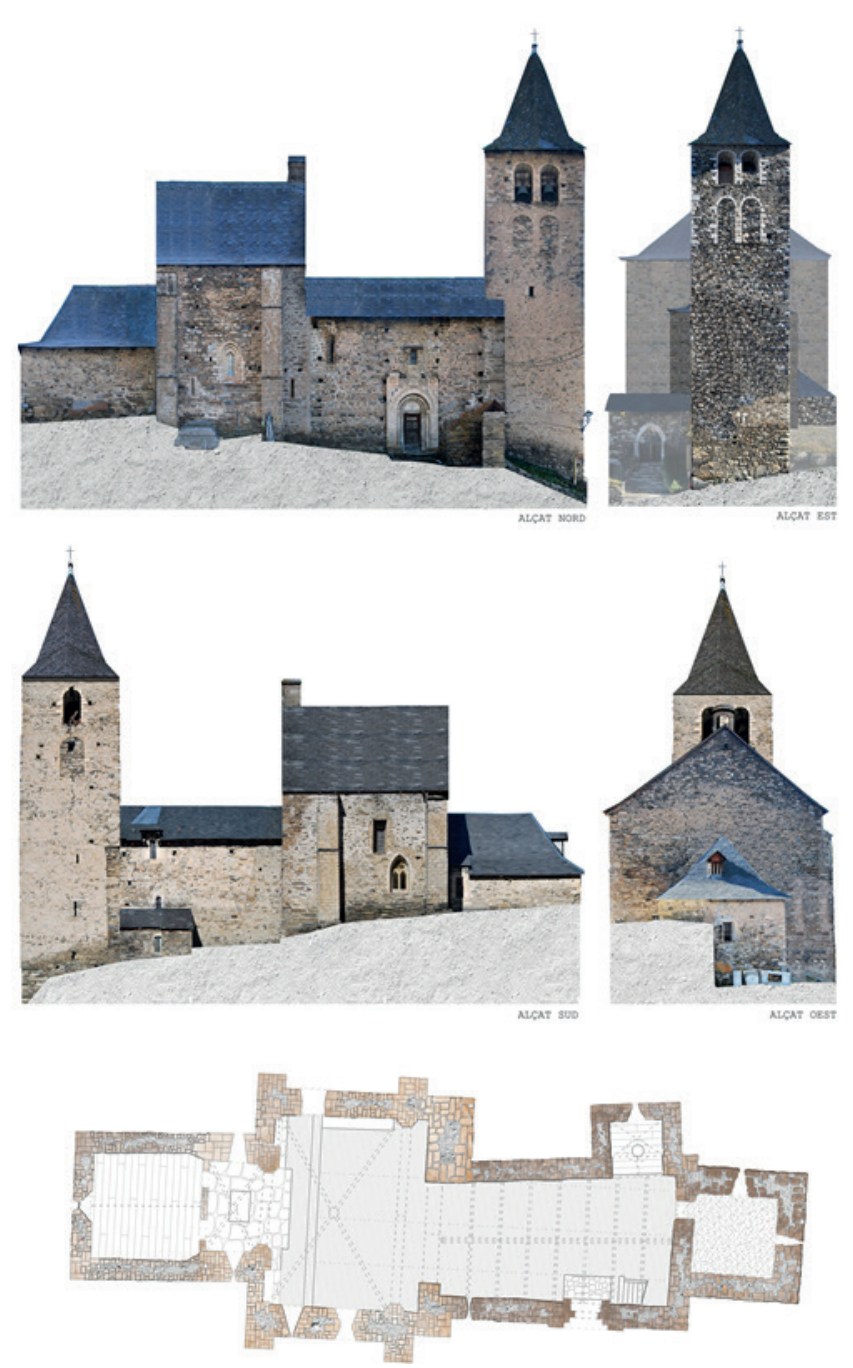

Fig. 2. Levantamiento de Sant Pèir d’Escunhau. Mónica López Piquer, Marta Gonzalvo Burcet y Helena Focadell Ciuret.

de los elementos verticales con el suelo del edificio. Lo representamos gráficamente por un plano $\left(\pi_{1}\right)$ que corta al ámbito $\left(A_{1}\right)$ en su punto de mayor cota topográfica $\left(a_{1}\right)$, en el intervalo $\left[a_{0}-a_{1}\right]$ desde la cota más baja del suelo. Esta área determinará el plano del De pavimento (Gem. Ani. L. 1, Cap. CXXXIV), mediante el cual podremos determinar las proporciones del Sancta y el Sancta Sanctorum.

Con el objeto de determinar la geometría de la sección se establecen unos planos $\left(\omega_{\mathrm{i}}\right)$ que son los transversales a la directriz $(\tau)$. Las secciones $\left(\omega_{\mathrm{i}}\right)$ variarán en función de la tipología de la planta, bien sea de planta basilical de tres naves o de nave única. Para determinar el estudio topológico se establecen unas secciones extremas, la $\left(\omega_{1}\right)$, la más próxima al ábside, y la $\left(\omega_{2}\right)$, en la fachada opuesta (Fig. 6). 

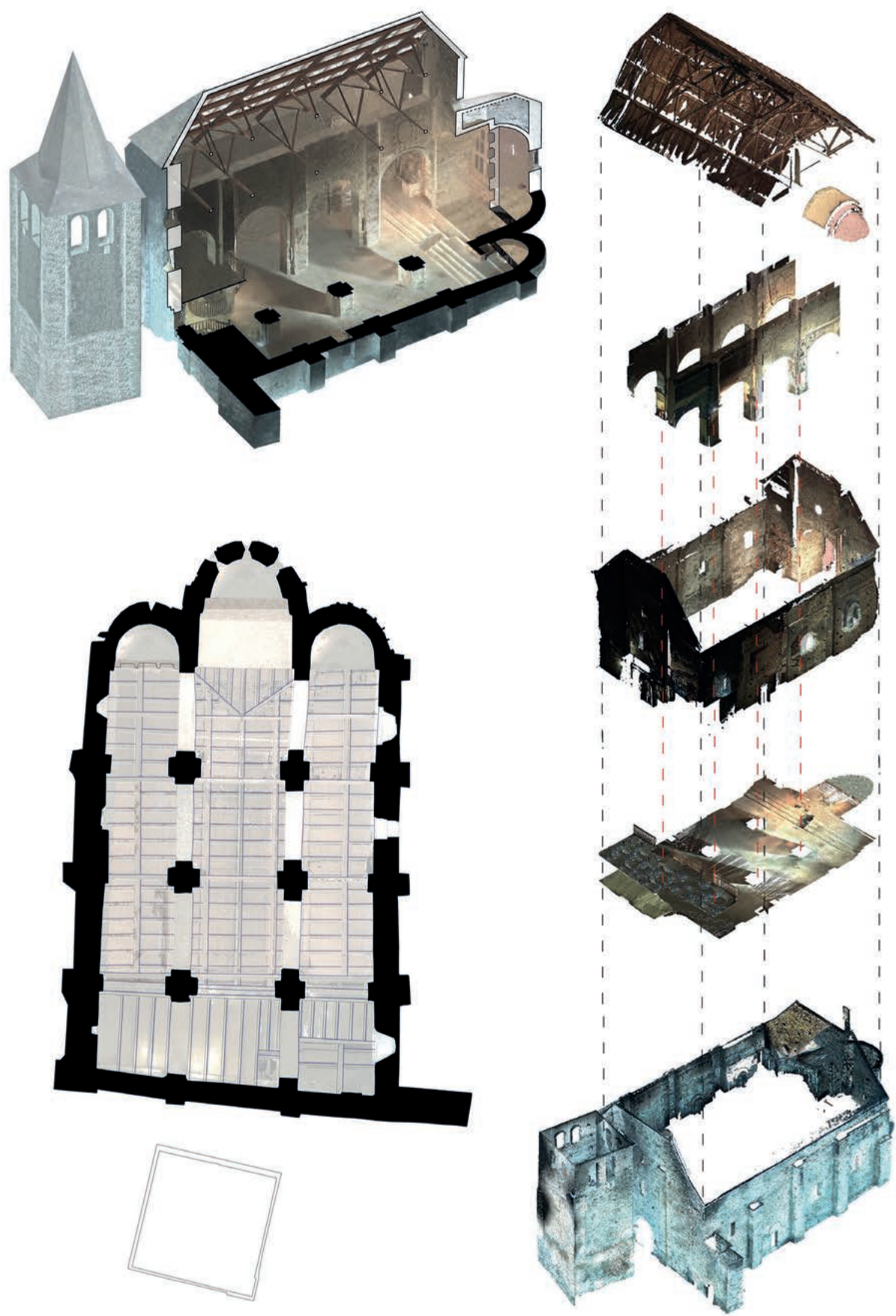

Fig. 3. Iglesia de Santa Maria de Cap d'Aran, Tredòs. 

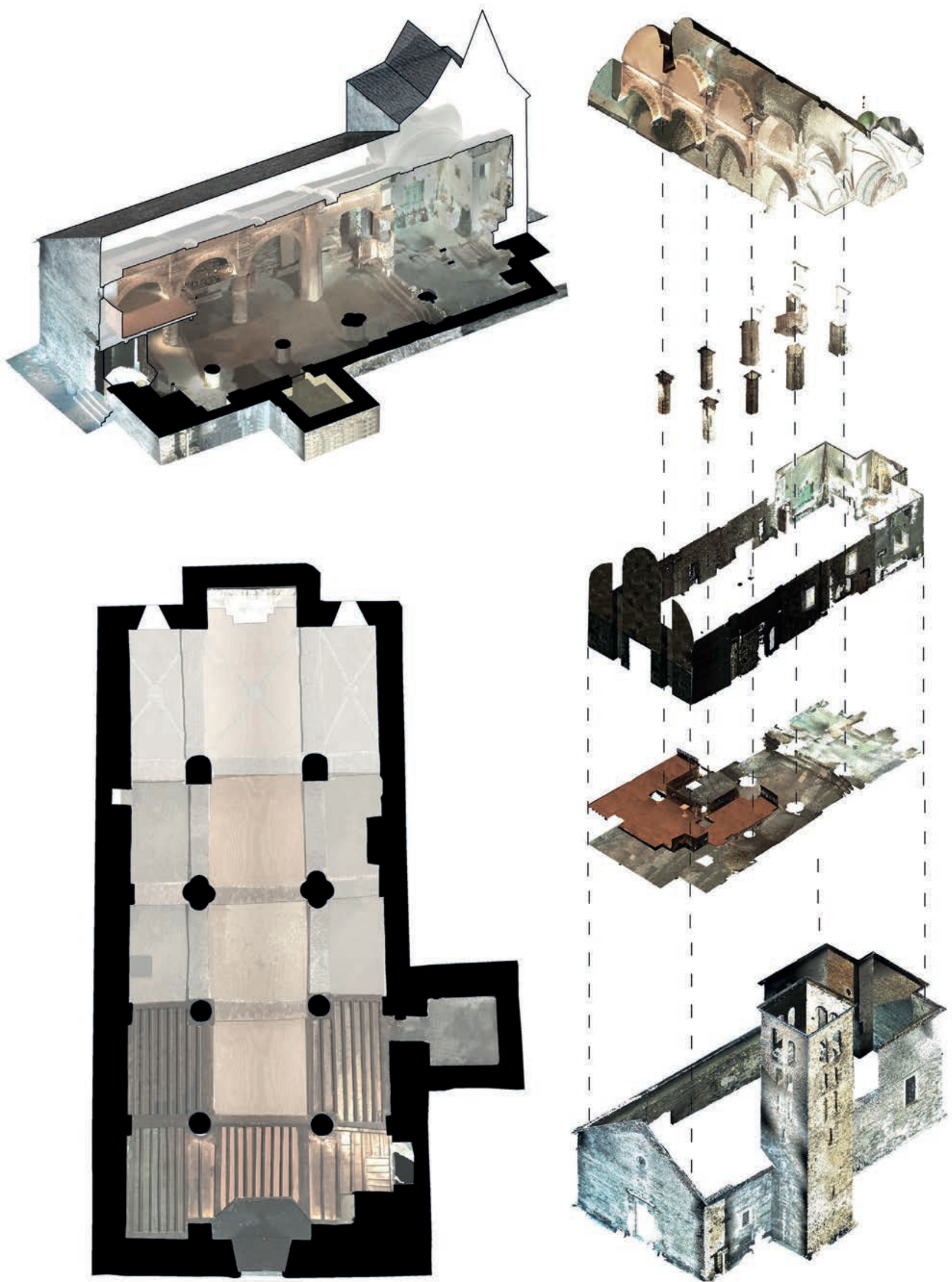

Fig. 4. Iglesia de Santa Maria de Vilamòs. 

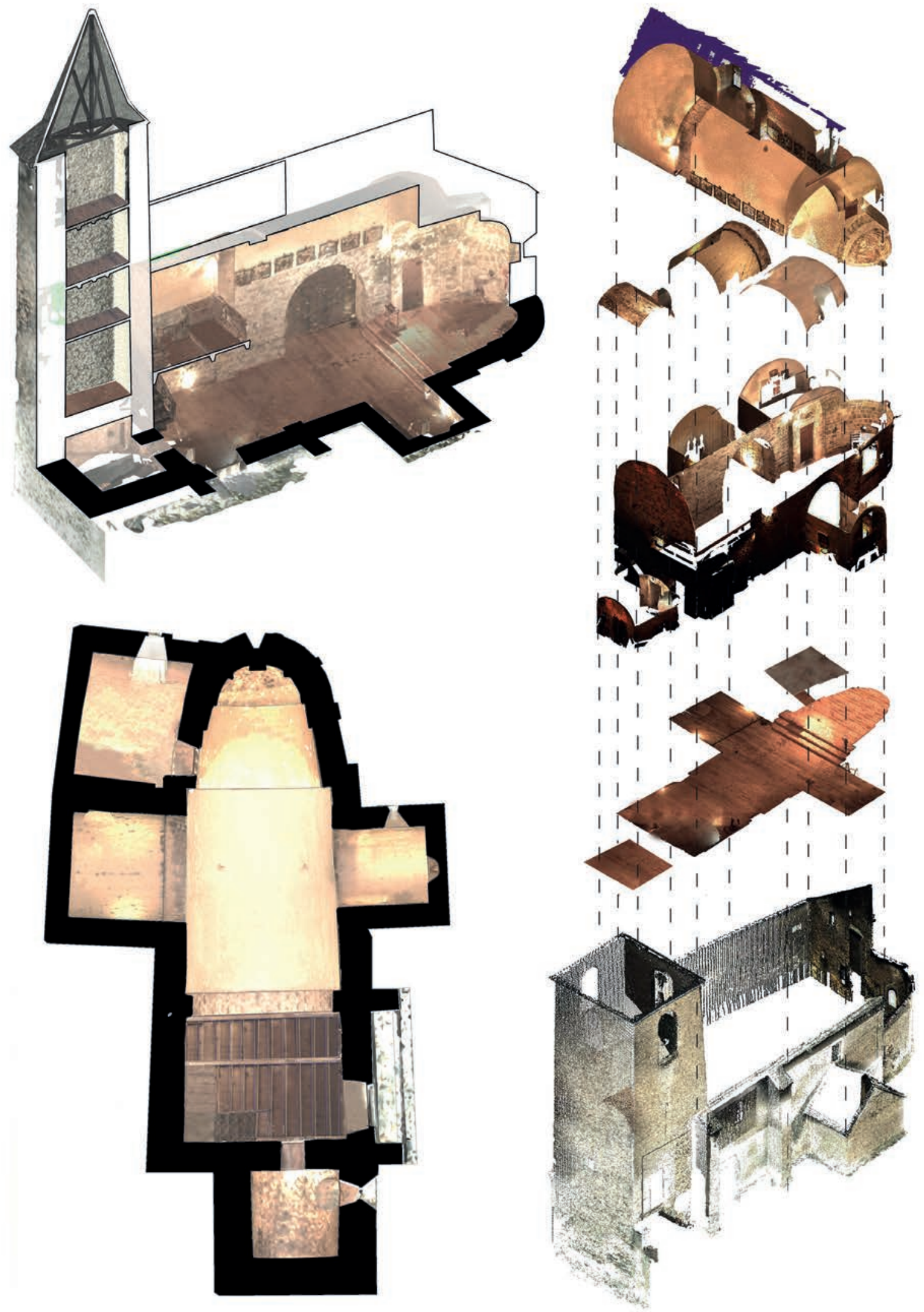

Fig. 5. Iglesia de Sant Pèir de Betlan. 




Sección horizontal A1

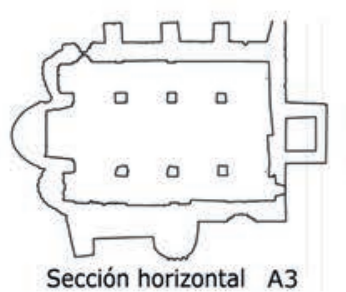

Sección horizontal A3

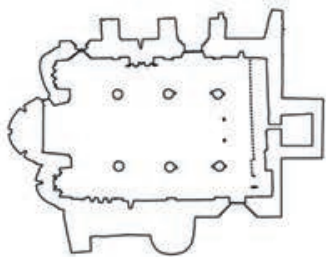

Sección horizontal A2

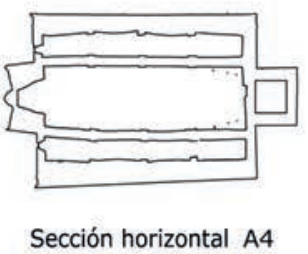

Sección horizontal A4

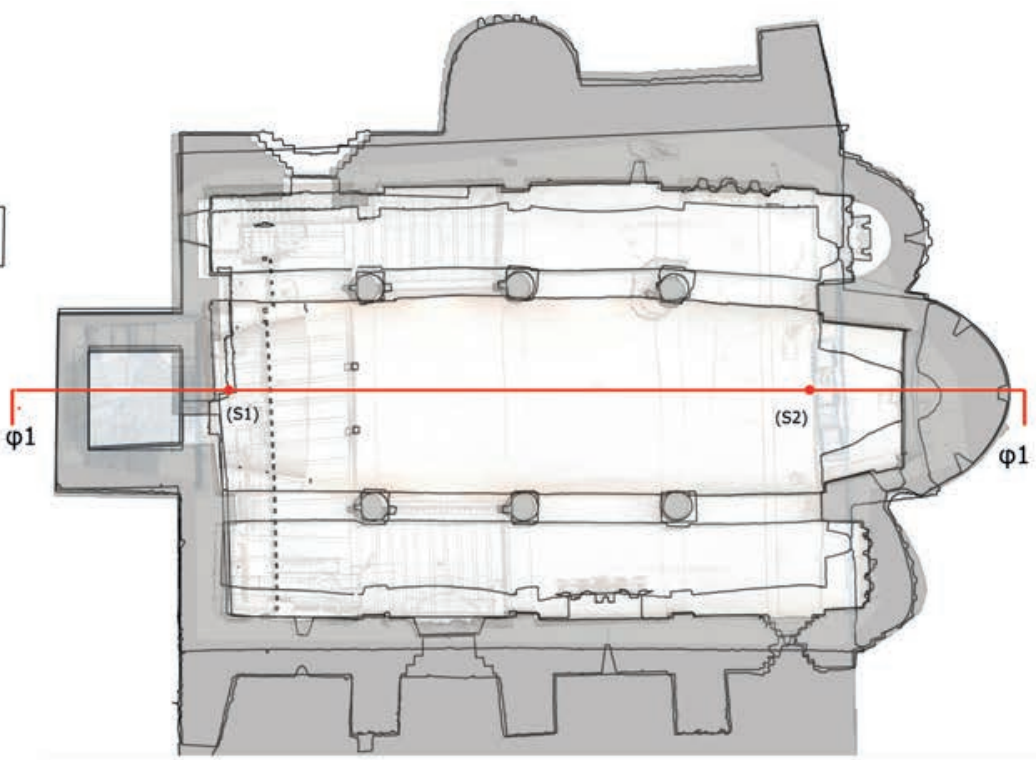

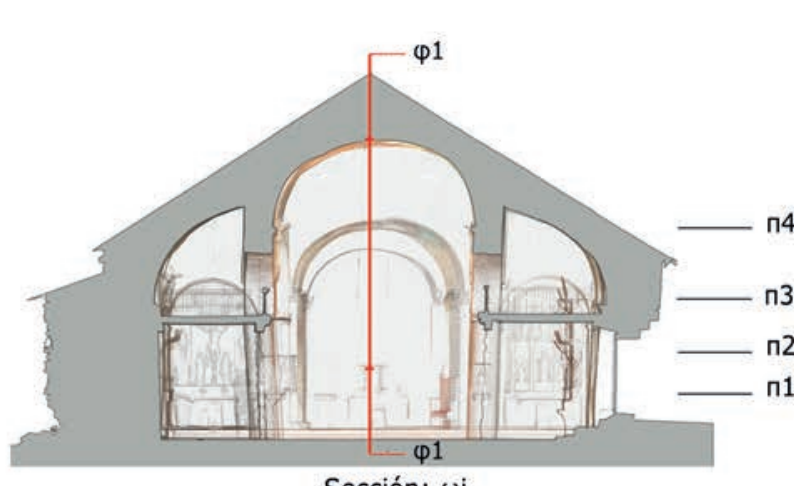

Sección: $\omega i$
(S1)

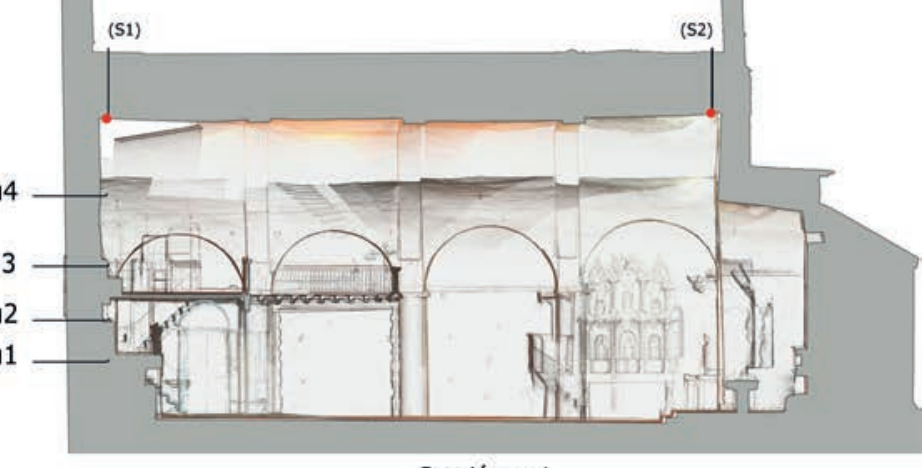

Sección: $\varphi 1$

Fig. 6. Metodología del análisis topológico.

\section{LA DIMENSIÓN DEL ESPACIO SAGRADO, EL TEMPLI Y LAS DE CAPELLIS}

Honorio de Autun avanza el futuro hacia el ideal gótico, defendiendo que el mundo es el templo de Dios. Estas construcciones románicas simbolizan las piedras reales del templo de gloria construido en la Jerusalén Celeste, venerando los antecedentes del Tabernáculo de Moisés y el Templo de Salomón (Gem. Ani. L. 1, Cap. CXXIII) (Mitra. L.1, Cap. XII). Dentro de esta clasificación, se pueden diferenciar entre las edificaciones que pueden ser definidas como Templi (Gem. Ani. L. 1, Cap. CXXVII) (Mitra. L.1, Cap. V) o Capellis (Gem. Ani. L. 1, Cap. CXXVIII) (Mitra. L.1, Cap. V). Las Capellis son construcciones más pequeñas e inicialmente itinerantes, instaladas en tiendas de piel de cabra, recibiendo este nombre de los capellanes, en referencia a la capa de San Martín, llamadas también capeum o lugar de limosna. Estas son las que, actualmente, se entenderían como ermitas (Monge España 2013). La diferencia de tipología, plantea el Templi con planta basilical con tres naves y las Capellis de nave única.

La definición topológica del espacio permite analizar en cada construcción el volumen útil interior $\left(\mathrm{V}_{\mathrm{u}}\right)$ en relación a la superficie interior $\left(\mathrm{S}_{\mathrm{u}}\right)$. Esto permite además determinar la utilización por habitante que pudieran tener estas construcciones. Con estos parámetros podremos determinar qué nivel de ocupación tienen tanto los Templi como las Capellis, con respecto a su volumen $\left(\mathrm{O}_{\mathrm{vu}}\right)$ y a su superficie $\left(\mathrm{O}_{\mathrm{su}}\right)$ (Tabla 2$)$. 


\begin{tabular}{|l|c|l|l|c|l|r|r|r|}
\hline Municipio & \multirow{2}{*}{ Fieles } & \multicolumn{1}{|c|}{ Nombre iglesia } & $\begin{array}{c}\text { Tipología } \\
\text { Nave }\end{array}$ & $\mathbf{V}_{\mathbf{u}}\left(\mathbf{m}^{3}\right)$ & $\mathbf{V}_{\mathbf{u}}$ Sancta & $\mathbf{S}_{\mathbf{u}}\left(\mathbf{m}^{2}\right)$ & $\mathbf{O}_{\mathbf{v u}}$ & $\mathbf{O}_{\text {su }}$ \\
\hline Tredós & 328 & Santa Maria de Cap d'Aran & Templi & $2.604,83$ & modificado & 305,32 & 7,94 & 0,93 \\
\hline Tredós & 328 & Sant Estèue de Tredòs & Capellis & 374,56 & modificado & 66,62 & 1,14 & 0,20 \\
\hline Unha & 177 & Santa Eulària d'Unha & Templi & 899,36 & original & 202,22 & 5,08 & 1,14 \\
\hline Escunhau & 213 & Sant Pèir d'Escunhau & Capellis & $1.113,37$ & modificado & 157,77 & 5,23 & 0,74 \\
\hline Vielha & 832 & Santa Maria de Mijaran & Templi & en ruinas & modificado & en ruinas & en ruinas & en ruinas \\
\hline Betlan & 156 & Sant Pèir de Betlan & Capellis & 478,96 & original & 99,85 & 3,07 & 0,64 \\
\hline Arròs & 208 & Sant Joan d'Arròs e Vila & Capellis & 212,66 & original & 64,74 & 1,02 & 0,31 \\
\hline Vilamòs & 208 & Santa Maria de Vilamòs & Templi & $1.290,27$ & original & 217,08 & 6,20 & 1,04 \\
\hline Vilamòs & 208 & Sant Miquèu de Vilamòs & Capellis & 125,18 & original & 34,64 & 0,60 & 0,17 \\
\hline Begòs & 104 & Sant Ròc de Begòs & Capellis & 232,00 & original & 80,69 & 2,23 & 0,78 \\
\hline Arres de Jos & 208 & Sant Fabian d'Arres de Jos & Capellis & 195,36 & modificado & 49,90 & 0,94 & 0,24 \\
\hline
\end{tabular}

Tabla 2. Relación entre el volumen interior $\left(\mathrm{V}_{\mathrm{u}}\right)$, superficie útil interior $\left(\mathrm{S}_{\mathrm{u}}\right)$ y el número de fieles.

\section{LA ORIENTACIÓN, ORTUM SOLIS AEQUINOCTIALEM}

Tanto las orientaciones de las iglesias como la colocación del altar y la sede, fueron evolucionando desde una orientación de los ábsides a poniente, a una alineación inversa, con el ábside al Este y, por ora parte, desde una alineación del altar y la sede determinada, a la colocación trasladada de la sede hacia la epístola. La cronología que determina las orientaciones de las iglesias se enmarca dentro de lo que se conoce como el primer románico aranés (Vogel 1962).

Isidoro de Sevilla (c. 556-636), en el Originum sive etymologiarum libri viginti (c. 630) define los De aedificiis sacris, de forma que los grandes edificios, templa, han de estar dispuestos en dirección al oriente equinoccial (Etymo. L XV. iii, iv). También lo hará Rabano Mauro (c. 776-856) en su De Universo libri viginti duo (c. 844), orientem expectabant cequinoctialem (Uni. L XIV. XXI). Esta tradición se impuso en las liturgias románicas con Honorio de Autun: ecclesiae ad orientem vertuntur ubi sol oritur (Gem. Ani. I, 129, De situ ecclesiae). Según Sicardo obispo de Cremona: Ad orientem, id est, ortum solis aequinoctialem (Mitra. I, 2, De fundatione ecclesiae). Para Jean Beleth: Versus orientem, hoc est, versus solis ortum aequinoctialem (Ration. cap. II, De loco). Guillermo de Durando: Yerus ortum solis aequinoctialem, ad denotandum quod ecclesia quae in terris militât (Prochi. I, 8, pp.5).

Una parte de la metodología de la arqueoastronomía actual, se basa en la astronomía moderna (GonzálezGarcía y Belmonte 2015). Ambas tienen como base universal, para la comparación de las orientaciones de los edificios, el concepto de declinación astronómica $(\delta)$. Este parámetro es independiente de las coordenadas terrestres y nos permite comparar la orientación de las iglesias estudiadas en territorios distantes. Se define como la proyección en la esfera celeste de la coordenada de la latitud terrestre y se obtiene a partir del acimut geográfico real (A), la altura del horizonte (h) y la latitud $(\phi)$.

En el periodo de este estudio, las hipótesis cultas de orientación son la canónica, de oriente a occidente, y la de la Festividad del Patrón de la advocación de la construcción. En cuanto a la primera tesis, hay que tener en cuenta que la declinación de un edificio orientado hacia el orto tiene un rango de coordenadas $\left[-23^{\circ} 26^{\prime},+323^{\circ} 26^{\prime}\right]$. Para los equinoccios astronómicos, la declinación es igual a $0^{\circ}$, mientras que en los solsticios de verano e invierno tienen sus valores extremos. Las orientaciones que no están en este rango, no están dentro del orto del horizonte. Para la segunda hipótesis de estudio, la de la fecha del Patrón de la construcción de la Iglesia, hay que tener en cuenta que el actual Calendario litúrgico se basa en el Calendario Romano de Pablo VI (1897-1978), el Mysterii paschalis celebrationem, y no coincide con los calendarios de referencia en los siglos XI-XII; el Martirologio Hieronymianum (s.VI) y, pudiéndose incorporar a finales del siglo XIII, la Legenda aurea de Jacopo della Voragine (1230-1298).

Para la determinación de la segunda hipótesis de estudio, la de la fecha del Patrón de la construcción, hay que tener en cuenta el cambio de la fecha del calendario Gregoriano (1582) de la bula Inter gravissimas del Papa Gregorio XIII (1502-1585), con el Juliano existente en el periodo de estudio. La Festividad del calendario eclesiástico tiene que tener en cuenta el desajuste del calendario Juliano con el Gregoriano. ${ }^{6}$ El ajuste de los calendarios de los equinoccios en el periodo de estudio (siglos XI-XIII), aequus nocte, era de entre cinco y siete días, donde el

\footnotetext{
Calculadora: https://carta-natal.es/calendario_gregoriano.php.
} 


\begin{tabular}{|c|c|c|c|c|c|c|c|c|c|c|c|c|c|c|c|c|c|c|c|c|}
\hline \multirow[b]{2}{*}{ Municipio } & \multirow[b]{2}{*}{ Iglesia } & \multicolumn{3}{|c|}{ Longitud } & \multicolumn{3}{|c|}{ Latitud $(\varphi)$} & \multirow[b]{2}{*}{$\begin{array}{l}\text { Hora } \\
\text { solar }\end{array}$} & \multirow{2}{*}{$\begin{array}{c}\text { Altura } \\
\text { (h) }\end{array}$} & \multirow{2}{*}{$\begin{array}{c}\text { Azimut } \\
\text { (A) }\end{array}$} & \multirow[b]{2}{*}{$\begin{array}{c}\text { Declin. } \\
\text { (ठ) }\end{array}$} & \multirow{2}{*}{$\begin{array}{c}\text { Día } \\
\text { 2016/17 }\end{array}$} & \multirow{2}{*}{$\begin{array}{l}\text { Des- } \\
\text { fase } \\
\text { días }\end{array}$} & \multirow{2}{*}{$\begin{array}{c}\text { Día } \\
1150\end{array}$} & \multirow{2}{*}{\multicolumn{2}{|c|}{$\begin{array}{c}\text { Fiesta Mayor/ } \\
\text { / Patrón Municipio }\end{array}$}} & \multirow{2}{*}{\multicolumn{2}{|c|}{$\begin{array}{c}\text { Calendario } \\
\text { Romano }\end{array}$}} & \multirow{2}{*}{$\begin{array}{l}\text { Des- } \\
\text { fase } \\
\text { días }\end{array}$} & \multirow{2}{*}{$\begin{array}{c}\text { Día } \\
1150\end{array}$} \\
\hline & & $\begin{array}{c}\begin{array}{c}\text { deci- } \\
\text { mal }\end{array} \\
\end{array}$ & $\mathbf{0}$ & ;' & decimal & o & , & & & & & & & & & & & & & \\
\hline Tredòs & anta Maria de Cap d'Aran & 0,92 & 0 & 55 & 42,70 & 42 & 42 & $7: 11$ & $32,40^{\circ}$ & $109,57^{\circ}$ & $-14,90^{\circ}$ & 9-Feb & -7 & 2-feb & 2 -ago & San Esteban I Papa & $V$ id. aug. & 10-ago & -7 & 3-ago \\
\hline Tredòs & Sant Estèue de Tredòs & 0,91 & 0 & 54 & 42,70 & 42 & 42 & $6: 48$ & $38,00^{\circ}$ & $102,14^{\circ}$ & $-9,30^{\circ}$ & 25 -Feb & -7 & 18-feb & 2-ago & San Esteban I Papa & ig. & $2-a \mathrm{a}$ & -7 & 26-jul \\
\hline Unha & Santa Eulària d'Unha &, 90 & 0 & 54 & 42,71 & 42 & 42 & $6: 18$ & $45,00^{\circ}$ & $92,58^{\circ}$ & $-2,29^{\circ}$ & 14-Mar & -7 & 7-mar & 10-dic & Santa Eulalia de Mérida & id. dic. & 10-dic & -7 & 3-dic \\
\hline Escunhau & Sant Pèir d'Escunhau & 0,83 & 0 & 49 & 42,70 & 42 & 41 & 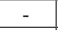 & $0,00^{\circ}$ & $50,90^{\circ}$ & $-47,30^{\circ}$ & \multicolumn{2}{|c|}{ solsticio verano } & - & 29-jun & San Pedro, apóstol & IIII n. iun. & 3-jun & -7 & 27-may \\
\hline Vielha & Santa María de Mijaran & 0,80 & 0 & 47 & 42,71 & 42 & 42 & $6: 37$ & $40,70^{\circ}$ & $98,73^{\circ}$ & $-6,59^{\circ}$ & 3-Mar & -7 & 24-feb & 29-sep & Sant Miquèu & IV id. aug. & 10-ago & -7 & 3-ago \\
\hline Betlan & Sant Pèir de Betlan & 0,79 & 0 & 47 & 42,73 & 42 & 43 & $6: 18$ & $45,00^{\circ}$ & $92,71^{\circ}$ & $-2,27^{\circ}$ & 14-Mar & -7 & 7-mar & 5-ago & F.M. Nòstra Sra. des Nhèus & III n. iun. & 3-jun & -7 & 27-may \\
\hline Arròs e Vila & Sant Joan d'Arròs e Vila & 0,77 & 0 & 46 & 42,76 & 42 & 45 & - & $0,00^{\circ}$ & $40,70^{\circ}$ & $-47,24^{\circ}$ & \multicolumn{2}{|c|}{ solsticio verano } & - & 29-ago & San Juan Bautista (Martirio) & VIII k. aug. & 25-ago & -7 & 18-ago \\
\hline Vilamòs & de Vilamòs & 0,73 & 0 & 43 & 42,75 & 42 & 44 & $6: 58$ & $35,40^{\circ}$ & $105,23^{\circ}$ & $-11,85^{\circ}$ & 18 -Feb & -7 & 11-feb & 15-ago & Asunción de la Virg & IV id. aug. & 10-ago & -7 & 3-ago \\
\hline Vilamòs & Sant Miquèu de Vilamòs & 0,74 & 0 & 44 & 42,75 & 42 & 44 & $6: 09$ & $46,90^{\circ}$ & $90,00^{\circ}$ & $-0,35^{\circ}$ & 19-Mar & -7 & 12-mar & 15-ago & Asunción de la Virgen María & IV id. aug. & 10-ago & -7 & 3-ago \\
\hline Begòs & Sant Ròc de Begòs & 0,74 & 0 & 44 & 42,74 & 42 & 44 & $7: 02$ & $34,40^{\circ}$ & $107,08^{\circ}$ & $-12,86^{\circ}$ & $15-\mathrm{Feb}$ & -7 & - & 16-ago & San Roque & - & - & -7 &  \\
\hline Jos & Sant Fabian d'Arres de Jos & 0,71 & 0 & 42 & 42,75 & 42 & 45 & 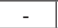 & $0,00^{\circ}$ & $164,72^{\circ}$ & $-47,25^{\circ}$ & \multicolumn{2}{|c|}{ solsticio invierno } & - & 20-ene & San Fabián Papa & k. ian & 1-ene & -7 & 25-dic \\
\hline
\end{tabular}

Tabla 3. Orientación Iglesias románicas del Val d’Aran.

ecuador celeste es paralelo a la declinación del Sol. En este entorno, podemos determinar los resultados de la investigación según los datos de la Tabla 3.

\section{EL ESPACIO SAGRADO, EL SANCTA Y EL SANCTA SANCTORUM}

El espacio definido por el Sancta y el Sancta Sanctorum tiene su referencia en el Tabernáculo de Moisés, referido por Honorio de Autum (Gem. Ani. L. 1, Cap. CXXIV, Cap. CXXIX) y por Sicardo de Cremona (Mitra, L. 1, Cap. 1). Era una tienda cuadrangular, de treinta codos de largo por diez de ancho, de proporción (1:3), por diez codos de alto de proporción en sección (1:1), ad quadratum. La tienda estaba dividida en dos compartimentos, el Sancta de 10 codos por 20 de proporción (1:2) dupla, y el Sancta Sanctorum, constituido por un cubo de 10 codos por lado, de proporción $(1: 1)$. El tabernáculo estaba rodeado por un atrio de 50 codos de ancho por 100 de largo de proporción (1:2). Ambos autores definen el Templo de Salomón como una reinterpretación del Tabernáculo (Gem. Ani. L. 1, Cap. CXXV; Mitra. L. 1, Cap. 1). El templo tenía sesenta codos de largo por veinte de ancho, con una proporción total de (1:3), y treinta codos de alto, con una proporción $(1: 1,5)$. El Templo disponía de un vestíbulo o entrada, de veinte codos de ancho por diez de profundidad, de proporción (1:0,5). Se accedía al Sanc$t a$, de veinte codos de ancho por cuarenta de largo, de relación (1:2) y a continuación al Sancta Sanctorum, un cubo de veinte codos por lado, con una proporción de $(1: 1)$ y una sección $(1: 1)$ (Fig. 7). Existen otros autores como Raoul de Saint-Trond (c. 1070-1138) en su Gesta abbatum Trudonensium (c. 1110-1120), que buscaba una relación antropomórfica, y entendía que, en su abadía, la construcción de su iglesia era como la de las iglesias que los entendidos consideran bien acabadas, porque estaba realizada a semejanza del cuerpo humano. (Continuatio III. Pars. I, col. 318C).

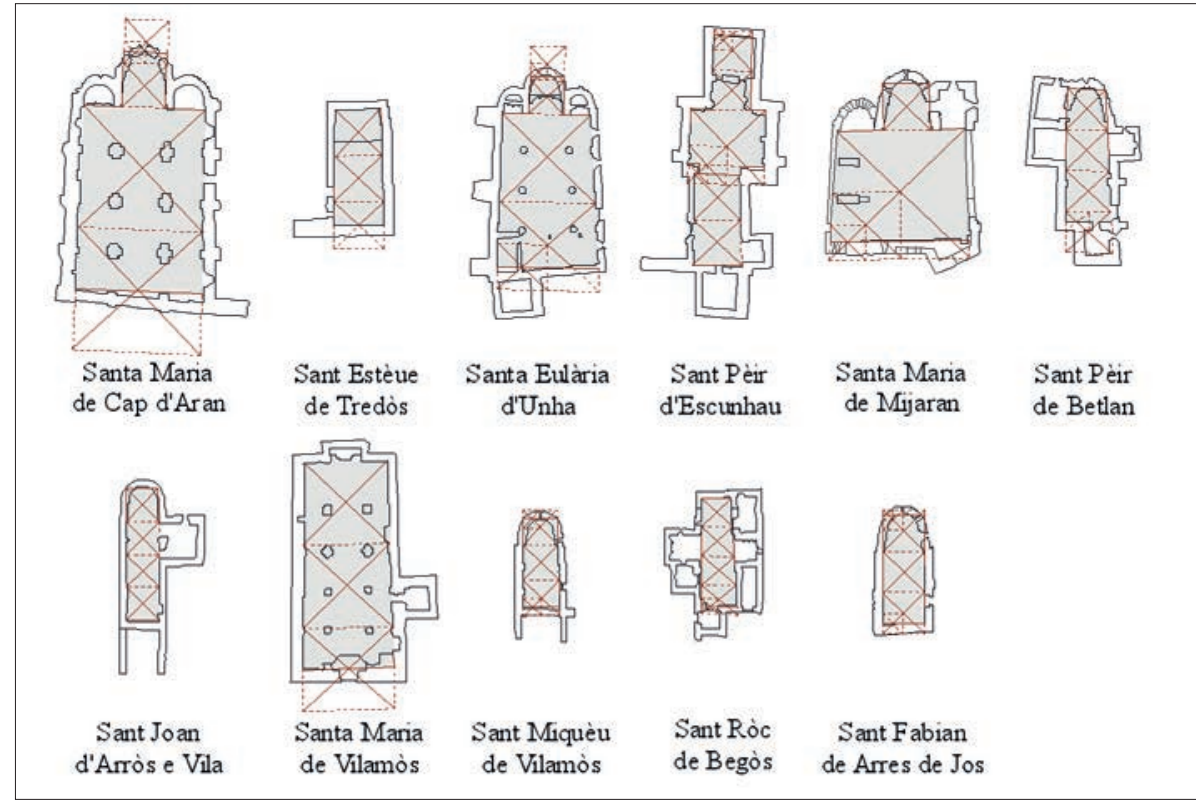

Fig. 7. Proporciones, en planta, de los espacios sagrados. 
La comparativa de las medidas y proporciones de los espacios sagrados, la realizaremos a través de la diferenciación entre las dos partes principales de los edificios, las cuales son el Sancta y el Sancta Sanctorum. Los datos analizados son la envergadura de los espacios, definidos como longitud, $\left(\mathrm{E}_{\mathrm{s}}\right)$ y $\left(\mathrm{E}_{\mathrm{ss}}\right)$, respectivamente, así como la luz total de los espacios, definida como anchura $\left(\mathrm{L}_{\mathrm{s}}\right)$ y $\left(\mathrm{L}_{\mathrm{ss}}\right)$. Estos datos están representados en la Tabla 4.
Según la metodología explicada anteriormente en la Figura 6, se han realizado 78 secciones tipo $\left(\omega_{i}\right)$. En ellas se determina previamente la proporción del cuerpo central de la edificación $\left(L_{\mathrm{sc}}\right)$, sea ésta la bóveda única, o la de la nave central, con el gálibo total de esta edificación $(a)$, que es la altura máxima de esta en la sección $\left(\omega_{\mathrm{i}}\right)$, a través de la relación $\left(a / L_{\mathrm{s}}\right)$ (Tabla 5 ; Fig. 8).

\begin{tabular}{|c|c|c|c|c|c|c|c|c|c|c|}
\hline \multirow[b]{2}{*}{ Municipio } & \multirow[b]{2}{*}{ Nombre Iglesia } & \multicolumn{3}{|c|}{ Proporción Sancta } & \multicolumn{3}{|c|}{ Proporción Sancta Sanctorum } & \multicolumn{3}{|c|}{ Proporción Total } \\
\hline & & $\mathbf{E}_{\mathrm{s}}(\mathbf{m})$ & $\mathbf{L}_{\mathrm{s}}(\mathbf{m})$ & $\begin{array}{l}\text { Proporción } \\
\text { Sancta }\end{array}$ & $\mathbf{E}_{\mathrm{ss}}(\mathbf{m})$ & $\mathbf{L}_{\mathrm{ss}}(\mathrm{m})$ & $\begin{array}{c}\text { Proporción } \\
\text { S. Sanctorum }\end{array}$ & $E_{t}(m)$ & $\mathbf{L}_{t}(\mathbf{m})$ & $\begin{array}{c}\text { Proporción } \\
\text { Total }\end{array}$ \\
\hline Tredós & Santa Maria de Cap d'Aran & 20,91 & 14,11 & 1,48 & 5,47 & 4,87 & 1,12 & 26,38 & 14,11 & 1,87 \\
\hline Tredós & Sant Estèue de Tredòs & 9,22 & 5,42 & 1,70 & 3,36 & 5,22 & 0,64 & 12,58 & 5,42 & 2,32 \\
\hline Unha & Santa Eulària d'Unha & 16,92 & 11,00 & 1,54 & 4,21 & 3,74 & 1,13 & 21,13 & 11,00 & 1,92 \\
\hline Escunhau & Sant Pèir d'Escunhau & 17,15 & 5,38 & 3,19 & modif. & modif. & - & modif. & modif. & - \\
\hline Vielha & Santa Maria de Mijaran & 12,07 & 15,39 & 0,78 & 5,47 & 5,44 & 1,01 & 17,55 & 15,39 & 1,14 \\
\hline Betlan & Sant Pèir de Betlan & 10,93 & 5,04 & 2,17 & 3,80 & 3,93 & 0,97 & 14,73 & 5,04 & 2,92 \\
\hline Arròs & Sant Joan d'Arròs e Vila & 10,94 & 3,52 & 3,11 & 3,93 & 3,42 & 1,15 & 14,88 & 3,52 & 4,23 \\
\hline Vilamòs & Santa Maria de Vilamòs & 16,89 & 10,01 & 1,69 & modif. & modif. & - & modif. & modif. & - \\
\hline Vilamòs & Sant Miquèu de Vilamòs & 6,96 & 3,95 & 1,76 & 2,79 & 3,28 & 0,85 & 9,75 & 3,95 & 2,47 \\
\hline Begòs & Sant Ròc de Begòs & 7,56 & 3,91 & 1,93 & 4,02 & 3,65 & 1,10 & 11,58 & 3,91 & 2,96 \\
\hline Arres & Sant Fabian de Arres de Jos & 10,18 & 4,52 & 2,25 & 1,89 & 3,50 & 0,54 & 12,08 & 4,52 & 2,67 \\
\hline
\end{tabular}

Tabla 4. Relación entre la planta del Sancta y el Sancta Sanctorum.

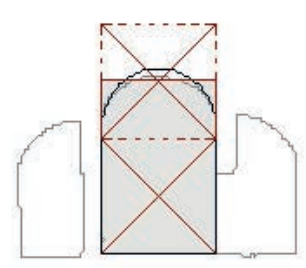

Santa Eulària d'Unha

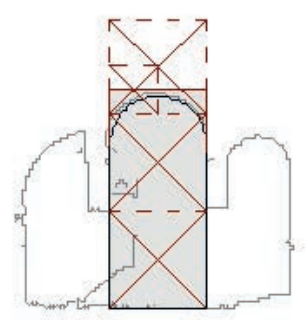

Santa Maria de Vilamòs

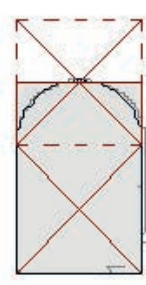

Sant Pèir de Betlan

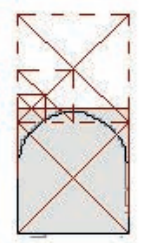

Sant Miquèu de Vilamòs

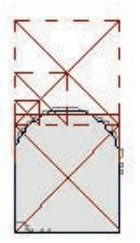

Sant Joan d'Arròs e Vila

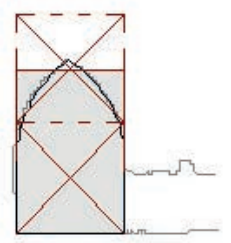

Sant Ròc de Begòs
Fig. 8. Proporciones, en sección, de los espacios sagrados. 


\begin{tabular}{|c|c|c|c|c|c|c|c|c|c|c|c|c|}
\hline \multirow{3}{*}{\begin{tabular}{c|} 
Municipio \\
Nombre \\
Iglesia \\
Sección $\left(\omega_{1}\right)$
\end{tabular}} & \multirow{2}{*}{\multicolumn{2}{|c|}{$\begin{array}{c}\text { Unha } \\
\begin{array}{c}\text { Santa Eulària } \\
\text { d'Unha }\end{array} \\
\end{array}$}} & \multirow{2}{*}{\multicolumn{2}{|c|}{$\begin{array}{c}\text { Betlan } \\
\text { Sant Pèirde } \\
\text { Betlan }\end{array}$}} & \multirow{2}{*}{\multicolumn{2}{|c|}{$\begin{array}{c}\text { Arròs e Vila } \\
\text { Sant Joan } \\
\text { d'Arròs e Vila }\end{array}$}} & \multicolumn{4}{|c|}{ Vilamòs } & \multirow{2}{*}{\multicolumn{2}{|c|}{$\begin{array}{c}\text { Begòs } \\
\begin{array}{l}\text { Sant Ròc de } \\
\text { Begòs }\end{array}\end{array}$}} \\
\hline & & & & & & & \multicolumn{2}{|c|}{$\begin{array}{l}\text { Santa Maria } \\
\text { de Vilamòs }\end{array}$} & \multicolumn{2}{|c|}{$\begin{array}{l}\text { Sant Miquèu de } \\
\text { Vilamòs }\end{array}$} & & \\
\hline & $\mathrm{a}(\mathrm{m})$ & $\mathrm{L}_{\mathrm{s}}(\mathrm{m})$ & $\mathrm{a}(\mathrm{m})$ & $\mathrm{L}_{\mathrm{s}}(\mathrm{m})$ & $\mathrm{a}(\mathrm{m})$ & $\mathrm{L}_{\mathrm{s}}(\mathrm{m})$ & $\mathrm{a}(\mathrm{m})$ & $\mathrm{L}_{\mathrm{s}}(\mathrm{m})$ & $\mathrm{a}(\mathrm{m})$ & $\mathrm{L}_{\mathrm{s}}(\mathrm{m})$ & $\mathrm{a}(\mathrm{m})$ & $\mathrm{L}_{\mathrm{s}}(\mathrm{m})$ \\
\hline$\omega_{1}$ & 6,42 & 3,98 & 6,71 & 4,41 & 4,33 & 3,69 & 7,38 & 3,37 & 4,25 & 3,81 & 6,02 & 3,81 \\
\hline$\omega \mathrm{v}_{2,1}$ & 6,36 & 4,12 & 7,13 & 4,60 & 4,53 & 3,73 & 7,62 & 3,45 & 4,46 & 3,82 & 5,96 & 3,84 \\
\hline$\omega \mathrm{v}_{1,1}$ & 6,68 & 4,18 & 7,18 & 4,86 & 4,54 & 3,69 & 7,90 & 3,56 & 4,52 & 3,85 & 5,78 & 3,85 \\
\hline$\omega \mathrm{v}_{2,2}$ & 6,65 & 4,28 & 7,19 & 4,63 & 4,52 & 3,74 & 7,81 & 3,67 & 4,56 & 3,87 & 5,89 & 3,92 \\
\hline$\omega \mathrm{p}_{1}$ & 7,11 & 4,24 & 7,15 & 4,33 & 4,28 & 3,17 & 7,40 & 3,05 & 4,34 & 3,75 & - & - \\
\hline$\omega \mathrm{v}_{2,3}$ & 7,20 & 4,73 & 7,31 & 4,81 & 4,57 & 3,69 & 7,59 & 3,75 & 4,50 & 3,89 & 5,79 & 3,86 \\
\hline$\omega \mathrm{v}_{1,2}$ & 7,10 & 4,66 & 7,32 & 4,92 & 4,57 & 3,67 & 7,53 & 3,78 & 4,49 & 3,83 & - & - \\
\hline$\omega \mathrm{v}_{2,4}$ & 7,19 & 4,73 & 7,33 & 5,03 & 4,60 & 3,66 & 7,55 & 3,69 & 4,50 & 3,94 & 5,77 & 3,95 \\
\hline$\omega \mathrm{p}_{2}$ & 6,69 & 4,52 & - & - & 4,49 & 3,58 & 7,33 & 7,46 & 4,29 & 3,82 & - & - \\
\hline$\omega \mathrm{v}_{2,5}$ & 7,19 & 4,96 & - & - & 4,70 & 4,58 & 7,57 & 3,68 & 4,72 & 3,89 & - & - \\
\hline$\omega \mathrm{v}_{1,3}$ & 7,27 & 4,98 & - & - & 4,58 & 4,58 & 7,45 & 3,66 & 4,72 & 3,87 & - & - \\
\hline$\omega \mathrm{v}_{2,6}$ & 7,26 & 5,07 & - & - & 4,75 & 4,56 & 7,59 & 3,64 & 4,72 & 3,90 & - & - \\
\hline$\omega \mathrm{p}_{3}$ & 6,39 & 4,81 & - & - & - & - & 7,39 & 3,32 & - & - & - & - \\
\hline$\omega \mathrm{v}_{2,7}$ & 7,36 & 5,20 & - & - & - & - & 7,41 & 3,60 & - & - & - & - \\
\hline$\omega \mathrm{v}_{1,4}$ & 7,25 & 5,22 & - & - & - & - & 7,43 & 3,62 & - & - & - & - \\
\hline$\omega \mathrm{v}_{2,8}$ & 7,25 & 5,21 & - & - & - & - & 7,61 & 3,62 & - & - & - & - \\
\hline$\omega_{2}$ & 7,29 & 5,22 & 7,27 & 5,10 & 4,83 & 3,44 & 7,55 & 3,68 & 4,67 & 3,89 & 5,78 & 3,85 \\
\hline Promedio & 6,98 & 4,71 & 7,18 & 4,74 & 4,56 & 3,83 & 7,54 & 3,80 & 4,52 & 3,86 & 5,86 & 3,87 \\
\hline $\mathbf{L}_{\mathrm{sc}}=\mathrm{a} / \mathrm{L}_{\mathrm{s}}$ & 1,48 & & 1,51 & & 1,19 & & 1,98 & & 1,17 & & 1,51 & \\
\hline
\end{tabular}

Tabla 5. Proporción de las secciones de la nave central

\begin{tabular}{|c|c|c|c|c|c|c|c|c|c|c|c|c|}
\hline \multirow{2}{*}{$\begin{array}{l}\text { Municipio } \\
\text { Nombre } \\
\text { Iglesia } \\
\end{array}$} & \multirow{2}{*}{\multicolumn{2}{|c|}{$\begin{array}{c}\text { Unha } \\
\text { Santa Eulària } \\
\text { d'Unha }\end{array}$}} & \multirow{2}{*}{\multicolumn{2}{|c|}{$\begin{array}{c}\text { Betlan } \\
\begin{array}{l}\text { Sant Pèir de } \\
\text { Betlan }\end{array} \\
\end{array}$}} & \multirow{2}{*}{\multicolumn{2}{|c|}{$\begin{array}{c}\text { Arròs e Vila } \\
\text { Sant Joan } \\
\text { d'Arròs e Vila } \\
\end{array}$}} & \multicolumn{4}{|c|}{ Vilamòs } & \multirow{2}{*}{\multicolumn{2}{|c|}{$\begin{array}{c}\text { Begòs } \\
\begin{array}{c}\text { Sant Ròc de } \\
\text { Begòs }\end{array}\end{array}$}} \\
\hline & & & & & & & \multicolumn{2}{|c|}{$\begin{array}{c}\text { Santa Maria de } \\
\text { Vilamòs } \\
\end{array}$} & \multicolumn{2}{|c|}{$\begin{array}{l}\text { Sant Miquèu de } \\
\text { Vilamòs } \\
\end{array}$} & & \\
\hline \multirow{2}{*}{ Sancta } & $\mathrm{a}(\mathrm{m})$ & $\mathrm{L}_{\mathrm{s}}(\mathrm{m})$ & $\mathrm{a}(\mathrm{m})$ & $\mathrm{L}_{\mathrm{s}}(\mathrm{m})$ & $\mathrm{a}(\mathrm{m})$ & $\mathrm{L}_{\mathrm{s}}(\mathrm{m})$ & $\mathrm{a}(\mathrm{m})$ & $\mathrm{L}_{\mathrm{s}}(\mathrm{m})$ & $\mathrm{a}(\mathrm{m})$ & $\mathrm{L}_{\mathrm{s}}(\mathrm{m})$ & $\mathrm{a}(\mathrm{m})$ & $\mathrm{L}_{\mathrm{s}}(\mathrm{m})$ \\
\hline & 6,98 & 11,00 & 7,18 & 5,04 & 4,56 & 3,52 & 7,54 & 10,01 & 4,52 & 3,95 & 5,86 & 3,91 \\
\hline $\mathbf{L}_{\mathrm{sc}}=\mathbf{a} / \mathbf{L}_{\mathrm{s}}$ & \multicolumn{2}{|c|}{0,63} & \multicolumn{2}{|c|}{1,42} & \multicolumn{2}{|c|}{1,30} & \multicolumn{2}{|c|}{0,75} & \multicolumn{2}{|c|}{1,14} & \multicolumn{2}{|c|}{1,50} \\
\hline
\end{tabular}

Tabla 6. Proporción del Sancta.

También se analiza la luz total de construcción $\left(L_{\mathrm{s}}\right)$, entendida como la distancia interior de los muros de cerramiento y el gálibo $\left(a / L_{\mathrm{s}}\right)$ (Tabla 6$)$.

\section{LA CONSTRUCCIÓN DEL ESPACIO SAGRADO, MURI LAPIDUM CAEMENTATI SUNT RELIGIOSORUM.}

Según la trascendencia simbólica de la construcción de la ecclesia materialis, la iglesia se cierra mediante muros, que representan a los de la Jerusalén celestial (Gem. Ani. L.I. Cap. CLXXV). Estos simbolizan las cuatro partes del mundo, los cuatro eruditos, las cuatro virtudes establecidas. La fortaleza de la edificación representa también a esta virtud (Mitra. L.I. Cap. IV), como también a los cuatro evangelistas. Por ello en el Liber de Miraculis Sancti Iacobi (c. 1140), dice refiriéndose a la basílica de Santiago de Compostela, "en esta iglesia no hay ninguna grieta o rotura; está maravillosamente construida" (Cap. IX. 2. De ecclesiae mensura). Encontraremos el mismo simbolismo 


\begin{tabular}{|l|l|r|r|r|r|r|r|r|r|r|r|r|}
\hline Municipio & Nombre Iglesia & $\mathbf{S}_{\mathbf{c}}\left(\mathbf{m}^{2}\right)$ & $\mathbf{S}_{\mathbf{u}}\left(\mathbf{m}^{2}\right)$ & $\mathbf{S}_{\mathbf{u}} / \mathbf{S}_{\mathbf{c}}$ & $\mathbf{S}_{\mathbf{m}}\left(\mathbf{m}^{2}\right)$ & $\mathbf{S}_{\mathbf{p}}\left(\mathbf{m}^{2}\right)$ & $\mathbf{S}_{\mathbf{p}} / \mathbf{S}_{\mathbf{m}}$ & $\mathbf{S}_{\mathbf{m c}}\left(\mathbf{m}^{2}\right)$ & $\mathbf{S}_{\mathbf{m} \mathbf{0}}\left(\mathbf{m}^{2}\right)$ & $\mathbf{S}_{\mathbf{p}} / \mathbf{S}_{\mathbf{m} \mathbf{0}}$ & $\mathbf{S}_{\mathbf{c o}}\left(\mathbf{m}^{2}\right)$ & $\mathbf{S}_{\mathbf{u}} / \mathbf{S}_{\mathbf{c o}}$ \\
\hline Tredós & $\begin{array}{l}\text { Santa Maria de Cap } \\
\text { d'Aran }\end{array}$ & 434,53 & 305,32 & $\mathbf{0 , 7 0}$ & 129,21 & 12,53 & $\mathbf{0 , 1 0}$ & 6,28 & 122,94 & $\mathbf{0 , 1 0}$ & 428,26 & $\mathbf{0 , 7 1}$ \\
\hline Tredós & Sant Estèue de Tredòs & 111,63 & 66,62 & $\mathbf{0 , 6 0}$ & 45,01 & - & - & 6,71 & 38,31 & - & 104,92 & $\mathbf{0 , 6 3}$ \\
\hline Unha & Santa Eulària d’Unha & 286,93 & 202,22 & $\mathbf{0 , 7 0}$ & 84,71 & 2,80 & $\mathbf{0 , 0 3}$ & 25,10 & 59,61 & $\mathbf{0 , 0 5}$ & 261,83 & $\mathbf{0 , 7 7}$ \\
\hline Escunhau & Sant Pèir d'Escunhau & 253,33 & 157,77 & $\mathbf{0 , 6 2}$ & 95,56 & - & - & 73,37 & 22,19 & - & 179,96 & $\mathbf{0 , 8 8}$ \\
\hline Vielha & Santa Maria de Mijaran & 297,14 & 208,23 & $\mathbf{0 , 7 0}$ & 88,91 & - & - & 0,00 & 88,91 & - & 297,14 & $\mathbf{0 , 7 0}$ \\
\hline Betlan & Sant Pèir de Betlan & 165,01 & 99,85 & $\mathbf{0 , 6 1}$ & 65,16 & - & - & 35,43 & 29,73 & - & 129,58 & $\mathbf{0 , 7 7}$ \\
\hline Arròs & Sant Joan d'Arròs e Vila & 102,75 & 64,74 & $\mathbf{0 , 6 3}$ & 38,01 & - & - & 10,16 & 27,85 & - & 92,59 & $\mathbf{0 , 7 0}$ \\
\hline Vilamòs & Santa Maria de Vilamòs & 321,79 & 217,08 & $\mathbf{0 , 6 7}$ & 104,71 & 4,46 & $\mathbf{0 , 0 4}$ & 9,91 & 94,80 & $\mathbf{0 , 0 5}$ & 311,88 & $\mathbf{0 , 7 0}$ \\
\hline Vilamòs & Sant Miquèu de Vilamòs & 62,54 & 34,64 & $\mathbf{0 , 5 5}$ & 27,90 & - & - & 4,01 & 23,89 & - & 58,52 & $\mathbf{0 , 5 9}$ \\
\hline Begòs & Sant Ròc de Begòs & 128,61 & 80,69 & $\mathbf{0 , 6 3}$ & 47,93 & - & - & 34,32 & 13,61 & - & 94,30 & $\mathbf{0 , 8 6}$ \\
\hline $\begin{array}{l}\text { Arres de } \\
\text { Jos }\end{array}$ & $\begin{array}{l}\text { Sant Fabian d'Arres de } \\
\text { Jos }\end{array}$ & 81,81 & 49,90 & $\mathbf{0 , 6 1}$ & 31,91 & - & - & 0,00 & 31,91 & - & 81,81 & $\mathbf{0 , 6 1}$ \\
\hline
\end{tabular}

Tabla 7. Relación elementos estructurales.

en la mezcla para unir los muros. El caementum, está compuesto por cal, agua y arena; la cal es el fervor de la caridad, el agua el Espíritu Santo, la mezcla con la arena, la espiritualidad de lo terrenal, sin la cual no es posible que haya vida (Mitra. L.I. Cap. IV). Honorio de Autun anuncia que las piedras están cogidas con cemento, al igual que los fieles lo hacen con el amor (Gem. Ani. L.I. Cap. CXXIX). Aunque en De constructione ecclesiae, imponía límites éticos a la construcción. Decía que está bien edificar iglesias y decorar construcciones con vasijas, vestidos, ornamentos; pero es mucho mejor gastar este mismo dinero en provecho de los indigentes y enviar su fortuna a los tesoros celestes a través de las manos de los pobres, y preparar allí en el cielo un regalo no material, sino eterno ( Gem. ani. L.I, CLXXI).

El análisis de los sistemas constructivos, permite investigar la relación entre la superficie total de la construcción $\left(\mathrm{S}_{\mathrm{c}}\right)$ y su superficie útil $\left(\mathrm{S}_{\mathrm{u}}\right)$, así como también determinar la relación entre la superficie de muros $\left(\mathrm{S}_{\mathrm{m}}\right)$ y pilares $\left(\mathrm{S}_{\mathrm{p}}\right)$. Los muros originales de cerramiento $\left(\mathrm{S}_{\mathrm{mo}}\right)$ son, a la vez, muros estructurales que han ido reforzándose con la construcción de contrafuertes $\left(\mathrm{S}_{\mathrm{mc}}\right)$. Con la diferencia entre la superficie total de construcción y de los contrafuertes, podemos determinar la superficie de la construcción original $\left(\mathrm{S}_{\mathrm{co}}\right)$ (Tabla 7).

\section{DISCUSIÓN DE LOS RESULTADOS TOPOLÓGICOS}

El análisis conmensurable realizado en estos edificios, permite determinar relaciones entre diferentes parámetros de las edificaciones.

1. Dimensión del espacio sagrado, Templi y De capellis. Del conjunto de iglesias del Val d'Aran que forman parte del primer románico, el 36,36\% son de planta basilical con tres naves, Templi, mientras que el 63,64\% restante son de nave única, Capellis. En el año 1313, los Templi tienen una relación entre la superficie útil y el número de fieles cercana a $1 \mathrm{~m}^{2} /$ persona (1:1), la cual es coincidente con la ocupación que actualmente define la Normativa española de ocupación. ${ }^{7}$ Este es el caso de Santa Maria de Cap d'Aran de Tredòs, Santa Eulària d'Unha y Santa Maria de Vilamòs. En las Capellis, la relación entre la superficie útil y el número de fieles aumenta considerablemente. Se diferencian funcionalmente de los Templi de planta basilical, ya que la ocupación llega a aumentar un $30 \%$, como en el caso de Sant Pèir

\footnotetext{
Código Técnico de la Edificación. Documento Básico SI Seguridad en caso de incendio. Consulta 11-01-2017 http://www.codigotecnico.org/images/ stories/pdf/seguridadIncendio/DccSI.pdf
} 
d'Escunhau, Sant Pèir de Betlan y Sant Ròc de Begòs, y llega a cuadriplicarse en Sant Estèue de Tredòs, Sant Joan d'Arròs e Vila, Sant Miquèu de Vilamòs y Sant Fabian d'Arres de Jos.

En cuanto al volumen (Tabla 2), podemos establecer que el $54,55 \%$ de estas iglesias conservan el volumen original del Sancta. Será el caso de Santa Eulària d'Unha, Sant Pèir de Betlan, Sant Joan d'Arròs e Vila, Santa Maria de Vilamòs, Sant Miquèu de Vilamòs y Sant Ròc de Begòs. El 45,45\% de bóvedas restantes pudieron tener un colapso estructural y fueron sustituidas por la estructura de madera que tienen actualmente, excepto en el caso de la iglesia de Santa Maria de Mijaran, que fue dinamitada en la Guerra Civil Española (1936-1939). Por esta causa tienen el volumen interior del Sancta modificado, como es el caso de Santa Maria de Cap d'Aran, Sant Estèue de Tredòs, Santa Eulària d'Unha, la nave de Sant Pèir d'Escunhau y Sant Fabian d'Arres de Jos. Por el contrario, los Sancta Sanctorum se han visto menos afectados por estas modificaciones. Las alteraciones, como en Santa Maria de Vilamòs, donde la totalidad del presbiterio es reformado en el siglo XIX, se producen por motivos litúrgicos. Lo mismo ocurre con la ocupación, la relación volumen/fieles de los Templi es superior a $5 \mathrm{~m}^{3} / \mathrm{fiel}$, mientras que en las Capellis este parámetro se reduce considerablemente.

2. La orientación, Ortum solis aequinoctialem. De los once resultados obtenidos en el estudio de las orientaciones (Tabla 3), se puede determinar que, tanto Santa Eulària d'Unha, Sant Miquèu de Vilamòs, como Sant Pèir de Betlan, están dentro de los rangos de la orientación canónica de oriente a poniente, lo que representa el $27,27 \%$ de las iglesias. Otras tienden a estar orientadas al solsticio de verano, como Sant Pèir d'Escunhau y Sant Joan d'Arròs, coincidentes con la festividad del Patrono de la Iglesia, mientras que Sant Fabian d'Arres de Jos está orientado hacia el solsticio de invierno, coincidente con el día del antiguo Patrón del siglo XIII, el 25 de diciembre. Las iglesias de Santa Maria de Cap d'Aran de Tredòs, Sant Estèue de Tredòs y Santa Maria de Vilamòs también coinciden con la fecha del Patrón, lo que representa que a esta tipología pertenecen el $54,55 \%$ de las edificaciones. Existen unas iglesias que, hasta el momento, no hemos sabido encontrar un criterio claro de orientación, éstas corresponden al 18,18\% y son Santa Maria de Mijaran y Sant Roc de Begòs. Entre los resultados astrofísicos, podemos destacar como curiosidad que la orientación de Sant Pèir de Betlan, según sus coordenadas $[(\phi)$, $(\lambda)$ ], en su declinación astronómica $(\delta=-2,27)$, la altura máxima del sol este día es exactamente $45^{\circ}$. Forma así el triángulo rectángulo isósceles $\left(45^{\circ}-90^{\circ}-45^{\circ}\right)$ definido en el Timaeus de Platón (c. 429-437 a. C.), como el elemento básico de la geometría, al que le asignará, además, el mayor grado de belleza (Tim. 53, c-d).

3. El espacio sagrado, el Sancta y el Sancta Sanctorum. La proporción de la planta del Sancta Sanctorum, tanto del Tabernáculo como del Templo de Salomón, es (1:1). Esta relación sólo existe en las iglesias de Santa Maria de Mijaran y Sant Pèir de Betlan. La proporción mayoritaria de estas iglesias tiende a $(1: 1+1 / 12)$, cosa que ocurre en Santa Maria de Cap d'Aran, Sant Esteve de Tredòs, Sant Joan d'Arròs y en Sant Ròc de Begòs. En cuanto a las proporciones del Sancta, en relación a la dupla (1:2), tanto del canon del Tabernáculo como del Templi, tan sólo Sant Ròc de Begòs tiende a ella. Finalmente, de la proporción tripla (1:3), de la totalidad interior del espacio sagrado, tan solo la mantienen Sant Pèir de Betlan y Sant Ròc de Begòs (Tabla 4).

En cuanto a las proporciones de las secciones de la nave central respecto a su gálibo (Tabla 5), en Santa Maria de Vilamòs es (1:2), mientras que en Santa Eulària d'Unha, Sant Pèir de Betlan y Sant Ròc de Begòs es de $(1: 1+1 / 2)$. Finalmente, tanto en Sant Joan d'Arròs e Vila como en Sant Miquèu de Vilamòs las proporciones son $(1: 1+1 / 20)$.

En cuanto a las proporciones del volumen del Sancta, analizadas a través de las secciones transversales de estos edificios (Tabla 6), hay que destacar que tanto Sant Pèir de Betlan como Sant Ròc de Begòs tienden a la proporción del Templo de Salomón $(1: 1+1 / 2)$, mientras que las edificaciones de planta basilical tienen una sección más baja a la del Tabernáculo de Moisés. La proporción es sesquiáltera en Santa Eulària d'Unha (2/3) y sesquitercia en Santa Maria de Vilamòs (3/4).

4. La construcción del espacio sagrado, Muri lapidum caementati sunt religiosorum. El promedio de relación entre la superficie interior de estas iglesias $\left(\mathrm{S}_{\mathrm{u}}\right)$ y la total construida actual $\left(\mathrm{S}_{\mathrm{c}}\right)$ es de $\left(0,64 \mathrm{~m}^{2} / \mathrm{m}^{2}\right)$, lo que significa que el $36 \%$ de la superficie de la construcción actual está destinada a elementos estructurales. Por otra parte, la relación entre la superficie interior $\left(\mathrm{S}_{\mathrm{u}}\right)$ y la construida original $\left(\mathrm{S}_{\mathrm{co}}\right)$ es de $\left(0,72 \mathrm{~m}^{2} / \mathrm{m}^{2}\right)$, es decir, el $28 \%$ de la superficie construida original corresponde a elementos estructurales (Tabla 7). Algunas 
de estas iglesias del primer románico han aumentado considerablemente su superficie construida, debido a la construcción de contrafuertes, por motivos estructurales. Este es el caso de Sant Pèir d'Escunhau, con el aumento de superficie del 28,96\%, Sant Pèir de Betlan, con un $21,47 \%$ más de estructura y, finalmente, en Sant Ròc de Begòs con un incremento del 26,69\% de su superficie construida inicial.

\section{CONCLUSIÓN}

Podemos concluir que, tan solo las secciones de Sant Pèir de Betlan y Sant Ròc de Begòs, consideradas $\mathrm{Ca}$ pelli, tienden a la misma proporción que la del Templo de Salomón $(1: 1+1 / 2)$. Las iglesias mayores, Templi, de planta basilical, disponen de una ratio de ocupación para el fiel, por su superficie interior, similar a los parámetros funcionales definidos en la actualidad. La proporción de las basílicas que conservan su sección es sesquiáltera en Santa Eulària d'Unha y sesquitercia en Santa Maria de Vilamòs. Este hecho hace pensar en la influencia de la proporción del mundo clásico en estas iglesias de planta basilical. Esta proporción fue transmitida por los principales comentaristas medievales de Platón. Es el caso del Timaeus translatus commentarioque instructus de Calcidio (f. 350), y el Comentarii in Somnium Scipionis de Macrobio (f. 400), y el De Nuptiis Philologiae et Mercurii de Marciano Capella (f. 430).

La iglesia de Sant Pèir de Betlan tiene una proporción en planta de (1:3), como el Templo de Salomón, y a su vez tiene orientación canónica, de oriente a occidente.

Por ello, de las once iglesias del primer románico, tan solo la iglesia de Sant Pèir de Betlan tiene una tendencia hacia los tratados litúrgicos Gemma animae de Honorio de Autun y el Mitralis de Officio de Sicardo obispo de Cremona.

\section{ABREVIATURAS}

De $\operatorname{archi} .=$ Vitrubio, M. 1899. Vitruvii. De architectura

Libri Decem. Iterum edidit Valentinus Rose.

De Uni. = De Universo Libri Viginti Duo. Rabanus Maurus. MPL111.

Etymo. $=$ Isidori Hispalensis episcopi. Etymologiarum sive Originvm libri XX. W. M. Lindsay.

Gem. Ani. = Gemma Animae. Honorius Augustodunensis. Patrologia Latina. MPL172.
Mitra.$=$ De Mitrali Seu Tractatus De Officiis Ecclesiaticis. Sicardus Cremoniensis. Patrologia Latina. MPL213.

Prochi. $=$ Guillermo de Durando. 1775. Prochiron vulgo rationale divinorum officiorum. Auctore Gulielmo Durando. Matriti: Ex typographia Blasii Roman.

Ration. $=$ Joanne Beletho. Rationale divinorum officiorum. MPL 202.

\section{FUENTES}

Blume, F., Lachman, K. y Rudorff, A. 1848. Die Schriften der Römischen Feldmesser Herausgegeben und erläutert von von F. Blume, K. Lachmann, und A. Rudorff. Erster Band. Bei Georg Reimer, Berlin.

Cantor, M. 1875. Die römischen Agrimensoren und ihre Stellung in der Geschichte der Feldmesskunst. Eine historisch-mathematische Untersuchung von Dr. Moritz Cantor. Druk un verlag von B.G.Teubner, Leipzig.

Cantor, M. 1880. Vorlesungen über Geschichte der Mathematik, von Moritz Cantor Erster Band. Von den ältesten Zeiten bis zum Jahre 1200 n. Chr. Druk und Verlag von B.G. Teuberner, Leipzig.

Fita, P.F. 1882. Le Codex de Saint-Jaques-de-Compostelle. Liber de Miraculis S. Jacobi. Livre IV. Paris: Maisonneuve et Cie..

Gourdon, M. 1884. “À travers l'Aran. Itinéraires d'un touriste”, Revue de Comminges. Saint Gaudens, Tomo 1.

Guillermo de Durando 1775. Prochiron vulgo rationale divinorum officiorum. Auctore Gulielmo Durando. Ex typographia Blasii Roman, Matriti.

Hanssens, J. M. 1948-1950. Amalarii episcopi Opera liturgica omnia, 3 vols. Bibliotheca Apostolica Vaticana, Rome.

Higinio 1998. Hyginus et Siculus Flaccus. Opuscula Agrimensorum veterum. Traducción y comentarios de $\mathrm{M}^{\mathrm{a}}$ José Castillo. Universidad de la Rioja, Logroño.

Honorius Augustodunensis 1895. "Gemma Animae", Documenta Catholica Omnia. De Scriptoribus Ecclesiae Relatis. Migne JP. Patrologia Latina. MPL172, Col. 0541 - 0738B.

Innocenzo III. 2002. Il sacrosanto mistero dell'altare. De sacro altaris misterio. Libreria editrice vaticana, Città del Vaticano.

Isidoro de Sevilla 1919. Isidori Hispalensis episcopi. Etymologiarum sive Originvm libri XX. Recognovit brevique adnotatione critica instruxit W. M. Lindsay. Tomus II. E Typographeo Clarendoniano, Oxonii.

Joanne Beletho 1855. "Rationale divinorum officiorum". Documenta Catholica Omnia. De Scriptoribus Ecclesiae Relatis. Migne JP. Patrologia Latina. MPL 202, Col. 0165 - 0167.

Lavallée, J. 1847. Espagne. L'Univers ou Histoire et description de tous les peuples, vol. II. Firmin Didot, París.

Madoz, P. 1845. Diccionario Geográfico-Estadístico-Histórico de España y sus posesiones de Ultramar de Pascual. Tomo II. Madrid: Est. LiterarioTipografico de P. Madoz y L. Sagasati.

Pablo VI, 1969. Calendarium Romanum ex decreto Sacrosanti Decumenici Concilii Vaticani II instauratum aictoritate Pauli PP. VI promulgatum. Typis Polyglottis Vaticanis, Vaticano.

Rabanus Maurus. 1864. "De Universo Libri Viginti Duo. Documenta Catholica Omnia. De Scriptoribus Ecclesiae Relatis". Migne JP. Patrologia Latina, MPL111, Col. 0009 - 0614B.

Rodulfi Abbatis S. Trudonis. 1895. "Gesta abbatum Trudonensium. Documenta Catholica Omnia. De Scriptoribus Ecclesiae Relati”s. Migne JP. Patrologia Latina. MPL 173, Col. 33-434

Ruperti Tuitiensis 1967. Ruperti Tuitiensis. Liber de divinis officiis editit Hrabanus Haacke. Typographi Brepols, Turnholti.

Sicardus Cremoniensis Episcopus 1855. "De Mitrali Seu Tractatus De Officiis Ecclesiaticis". Documenta Catholica Omnia. De Scriptoribus Ecclesiae Relatis. Migne JP. Patrologia Latina, MPL213, Col. 0011 - 0011 
Smedt, C; Backer I (et alt). 1894. Praemissum est Martyrologium Hieronymianum endentibus Iohanne Baptista de Rossi et Ludovico Ouchesme. Sanctorum novembris collecta digesta illustrata. Tomi II pars prior: Apud socios Bollandianos, Bruxellis.

Viollet-le-Duc, E. E. 1972. Voyage aux Pyrénées 1833: lettres à son père et journal de route. Les Amis du Musée Pyrénéen, Lourdes.

Vitrubio, M. 1878. Los diez libros de archîitectura de M. Vitruvio Polión, traducidos del latin, y comentados por Don Joseph Ortiz i Sanz presbitro. Imprenta Real, Madrid.

Vitrubio, M. 1899. Vitruvii. De architectura Libri Decem. Iterum edidit Valentinus Rose. In aedibus B. G. Teubneri, Lipsiae.

Vorágine, J. (1844-1845). La leyenda de Oro para cada día del año. Vidas de todos los Santos que venera la Iglesia: Obra que comprende todo el Ribadeneyra mejorado, las noticias del Croisset, Butler, Godescard, la revisa José Palau. 4 Tomos. Librería de Razola, Imprenta de Llorens Hermanos, Madrid - Barcelona.

\section{BIBLIOGRAFÍA}

Alcolea, S. 2008. La missió arqueologica del 1907 als Pirineus. Fundació La Caixa, Barcelona.

Carrero Santamaría, E. 2009. "La arquitectura medieval al servicio de las necesidades litúrgicas. Los conjuntos de iglesias”, Anales de Historia del Arte, volumen extraordinario, pp. 61-97.

Durliat, M. y Allègre, V. 1969. Pyrénées Romanes. Zodiaque. La nuit des temps Colections, la-Pierre-Qui-Vire, Paris.

Español, F. 2013. "El Pirineu desvetllat: Viatges i descoberta del patrimoni medieval el pirineu desvetllat: Viatges i descoberta del patrimoni medieval", Annals del Centre d'Estudis Comarcals del Ripollès, Annals 20102011, IBIX 7, pp. 13-36.

Garland, E. 2012. "Les débuts de l'art roman dans le Val d'Aran", Mémoires de la Société Archéologique du Midi de la France, T. LXXII, pp. 82-105. Tolosa de Languedòc.

González-García, A. C. y Belmonte, J. A. 2015. "The Orientation of PreRomanesque Churches in the Iberian Peninsula", Nexus Network Journal, 17 (2), pp. 353-377.

Granell, E. y Ramon, A. 2006. Lluís Domènech i Muntaner: viatges per l'arquitectura románica. Col·legi d'Arquitctes de Catalunya, Barcelona.

Guarnieri, A., Vettore, A. y Remondino, F. 2004. "Photogrammetry and ground-based laser scanning: assessment of metric accuracy of the 3D model of Pozzoveggiani Church", en Working Week, The Olympic Spirit in Surveying. Athens.

Íñiguez, J. A. 1986. "La simbología del templo cristiano en el comienzo del periodo gótico (Honorio de Autún y Sicardo de Cremona). Biblia y hermenéutica”, en José María Casciaro (ed.), VII Simposio Internacional de Teología de la Universidad de Navarra, pp. 667-681. Servicio de Publicaciones de la Universidad de Navarra, Pamplona.

Lluis i Ginovart, J., Costa, A, y Coll, S. 2016a. Thrantem Lapides MMXVI. EAR, Universitat Rovira Virgili, Tarragona.

Lluis i Ginovart, J., Costa, A. y Coll, S. 2016b. Glèises en Encurnanclinc de voutes Esgarramingades. EAR, Universitat Rovira i Virgili, Institut Estudis Ilerdencs, Tarragona.

Mambelli, F. 2004. "Il problema dell'immagine nei commentari allegorici sulla liturgia: Dalla Gemma Animae di Onorio d'Autun (1120 ca.) al Rationale divinorum officiorum di Durando di Mende (1286-1292)", Studi Medievali, Jun., 45 I (1), pp. 121-158.

Martínez, A. N. 2002. "Cenobios leoneses altomedievales ante la europeización: San Pedro y San Pablo de Montes, Santiago y San Martín de Peñalba y San Miguel de Escalada”, Hispania Sacra, 54, 109, pp. 87-108, doi: https://doi.org/10.3989/hs.2002.v54.i109.189.

Mews, C. J. 2009. "Liturgists and Dance in the Twelfth Century: The Witness of John Beleth and Sicard of Cremona", Church History, 78 (3), September, pp. 512-548, doi: https://doi.org/10.1017/s0009640709990412.

Monge España, E. 2013. "Capèles romaniques d'origina romanica ena Val d'Aran". Era Batalha de Murèth, 1213. Era Queremònia, 1313. Era grana patzeria, 1513. Tres hites importantes entara Val d'Aran. Jornades d'estudi. Val d'Aran 18-20 de Octubre de 2013, pp. 111- 118. Salardú.

Pesci, A., Bonali, E., Galli, C. y Boschi, E. 2012. "Laser scanning and digital imaging for the investigation of an ancient building: Palazzo d'Accursio study case (Bologna, Italy)", Journal of Cultural Heritage, 13 (2), pp. 215-220, doi: https://doi.org/10.1016/j.culher.2011.09.004.

Pladevall, A. (dir.) 1987. Catalunya Romànica. El Solsonès. La Vall d'Aran, Vol. XIII. Enciclopèdia Catalana, Barcelona.

Polo, J. y Cots, P. 2009. "Santa Maria d'Arties (Naut Aran, Val d'Aran): Un exemple integrau a nivèu de restauracion d'un monument a compdar des entresenhes arqueologiques", en Cicle de conferències patrimoni arqueològic i arquitectònic a les Terres de Lleida 2009, pp. 75-103. Departament de Cultura i Mitjans de Comunicació.

Puig i Cadafalch, J. 1906. "Influences lombardes en Catalogne", en Congrès Archéologique de la France, Carcassonne-Perpignan, pp. 684-703.

Puig i Cadafalch, J., Falguera, A. de y Goday y Casals, J. 1918. L'Arquitectura Romànica a Catalunya. Vol III. Institut d'Estudis Catalans, Barcelona.

Reglà, J. 1948. "Cuestiones demográficas del valle de Arán en la Edad Media", Pirineos, 10, pp. 497-508.

Reglà, J. 1951. Francia, la corona de Aragón y la frontera pirenaica: la lucha por el valle de Arán, siglos XIII-XIV. CSIC, Madrid.

Reveyron, N. 2003. "Architecture, liturgie et organisation de l'espace ecclésial. Essai sur la notion d'espace dans l'architecture religieuse du Moyen Âge", Les cahiers de Saint-Michel de Cuxa, 34, pp. 161-175.

Ros Barbosa, E. 2015. "The movable and immovable heritage of the Aran Valley and its management”, en Proceedings of the Structural Studies, Repairs and Maintenance of Heritage Architecture XIV. Stremah, Section 8, pp. 469-480.

Sàez-Aragonès, G. 1976. "Primera fase del proyecto de restauración de la Iglesia de Santa María de Artíes en el Valle de Arán (Lérida)", Cuadernos de Arquitectura y Urbanismo, 116, pp. 63-71.

Sanllehy i Sabi, A. 1996. "Comunitats, veïns i arrendataris a la Val d'Aran (s. XVII-XVIII): Dels usos comunals a la dependencia económica”. Pedralbes, 16, pp. 189- 197.

Sarrate i Forga, J. 1975a. El Arte románico en el Cap d'Aran. Colegio Oficial de Aparejadores y Arquitectos de Cataluña, Delegación de Lérida.

Sarrate i Forga, J. 1975b. El Arte románico en el Mig-Arán. José Sarrate Forga, Lérida.

Sarrate i Forga, J. 1976a. El Arte románico en el Baix-Arán. José Sarrate Forga, Lérida.

Sarrate i Forga, J. 1976b. "Esquema del Arte Románico Aranés”. Cuadernos de Arquitectura y Urbanismo, 116, pp. 54-63.

Sebastián, S. 1994. Mensaje Simbólico del Arte Medieval. Arquitectura, Liturgia e Iconografia. Ediciones Encuentro, Madrid. pp. 352-355.

Vogel, C. 1962. "Sol aequinoctialis. Problemes et tecnique de l'orientation dans le cultura chretien", Revue Sciences Religieuses, 36, pp. 175-211, doi: https://doi.org/10.3406/rscir.1962.2332. 